\title{
On The Closed Form Strategies of an Investor under the CEV and CIR Processes
}

\author{
Edikan E. Akpanibah ${ }^{1,2}$, Bright O. Osu ${ }^{1,5^{*}}$, Everestus O. Eze ${ }^{1}$, \\ Chidi U. Okonkwo ${ }^{1,4}$, Ben I. Oruh ${ }^{1}$, Udeme O. Ini ${ }^{3}$
}

1. Department of Mathematics, Michael Okpara University of Agriculture, Umudike, Nigeria.

2. Department of Mathematics and Statistics, Federal University Otuoke, Bayelsa, Nigeria.

3. Department of Mathematics and Computer Science, Niger Delta University, Bayelsa, Nigeria.

4. Department of Mathematics and Statistics, Caritas University, Amorji Nike. Enugu, Nigeria.

5. Department of Mathematics, Abia State University, Uturu.

*Corresponding author: osu.bright@mouau.edu.ng

\section{Article Info}

Received: 01 June 2021

Accepted: 17 September 2021
Revised: 15 September 2021

Available online: 10 October 2021

\begin{abstract}
In this paper, the explicit solutions of the optimal investment plans of an investor with exponential utility function exhibiting constant absolute risk aversion (CARA) under constant elasticity of variance $(\mathrm{CEV})$ and stochastic interest rate is studied. A portfolio comprising of a risk-free asset modelled by the Cox-Ingersoll-Ross (CIR) process and two risky assets modelled by the CEV process is considered, where the instantaneous volatilities of the two risky assets form a $2 \times 2$ matrix $n=\left\{n_{p, q}\right\}_{2 \times 2}$ such that $n n^{T}$ is positive definite. Using the power transformation and change of variable approach with asymptotic expansion technique, explicit solutions of the optimal investment plans are found. Moreover, numerical simulations are used to study the effects of the interest rate, elasticity parameter, correlation coefficient and the risk averse coefficient on the optimal investment plans.
\end{abstract}

Keywords: Asymptotic technique, CEV process, Cox-Ingersoll-Ross process, Exponential utility, Optimal investment plan, Power transformation.

MSC2010:97M30, 98B26

\section{Introduction}

The optimal investment plan of utility maximization is a basic problem in the study of mathematical finance and has attracted attentions from a good number of authors which has led to numerous researches in this area. [1] used the optimal control method to study the optimal investment plan for the first time. [2] - [4] studied the problem of utility maximization using stochastic optimal control theory. Other authors such as [5] - [7], used the Martingale method to solve optimization problems related to optimal investment plan. $[3,8]$ studied the problem of utility maximization for an incomplete market. The optimal investment plan with stochastic interest rate under geometric 
Brownian motion (GBM) has been studied by some authors; these include [9], who studied the investment plan under stochastic interest rate for a case of protected defined contribution (DC) fund. [10] - [11] modelled the risk free interest rate using CIR process to obtain optimal investment plan for a DC plan. In [12]- [13], the risk free interest rate followed the Vasicek model. [14] - [16] studied the optimal investment plan when the interest rate is of affine type. However, all the authors above used the GBM to model the risky assets but [17] showed that the GBM process is not practical in real life since the volatilities of the stock market prices is assumed to be constant The importance of stochastic volatilities cannot be undermined as it plays a crucial role in the behaviour of the market prices of the risky assets due to its fluctuating nature resulting from various information available in the market especially now that financial institutions in most countries and even the financial markets are currently in serious crisis due to the disgusting effect of the novel corona virus (Covid-19) pandemic. To make a relatively near right decision during investment in assets such as stock, the stochastic volatility models become necessary to understand the fluctuating nature of the stock market price. In this research, the optimal investment plan is investigated for a case where the risky assets follow the CEV model and the risk free asset follows the CIR process. The CEV model is one of the stochastic volatility models used to describe the stock market price behaviours. It was first used in [18] and has the ability to capture the implied volatility skew.

A good number of researches have been done on optimal investment plan under the CEV model. [19] studied the optimal investment plan with dividend, taxes and transaction cost under the CEV model with different utility functions. [20] studied the optimal investment plan and reinsurance problem under the CEV process. [21] - [22] solved the optimal investment problem for a defined contribution (DC) pension plan with return of premiums clauses under different assumptions and assumed that the stock market price follows the CEV process. In all the literature above under CEV processes, the interest rates were assumed to be constant but however, there are some works under the CEV process that their interest rate are stochastic.

In [23], an investor's exponential utility was maximized for a case where the interest rate and stock market price was modelled by CIR and CEV process respectively. They used the Legendre transformation and asymptotic expansion method to determine an explicit solution of the optimal investment plan. They outlined the complexity involved in solving optimization problems that combined both CEV process and stochastic interest rate. Also, they pointed out that in real life applications, interest rates are usually not constant but fluctuating in nature and the volatility of the interest rate generate some market risks; that is to say, when these risks are not considered, we are under estimating the effect of this risk emanating from this interest rate which is critical in influencing the prices of different assets available in financial market. [24] studied the optimal investment plan with stochastic interest rate under the CEV model using logarithm utility; they considered investment in one risk free asset and a risky asset and assumed that the interest rate follows the Cox- Ingersoll-Ross (CIR) process. The power transformation, change of variable and asymptotic approach was used to determine the asymptotic solution of the optimal investment plan. [25] modelled the risky asset with modified CEV process and the interest rate with O-U process and determined the optimal investment plan for an investor with exponential utility. Also, an investor's investment plan with stochastic interest rate under the CEV model and the O-U Process was studied by [26]. In their work, they used two risky assets modelled by the CEV model and a risk free asset modelled by $\mathrm{O}-\mathrm{U}$ process and observed that the optimal investment plans exhibit a fluctuating effect.

In this paper, the expected exponential utility of an investor's terminal wealth is being maximized by studying the optimal investment plans of an investor exhibiting the CARA. Here, the two risky assets follow the CEV process while risk free interest rate follows the CIR process. More so, we use the power transformation, variable change and asymptotic method to determine asymptotic solutions of the optimal investment plan. The main difference between our work and that of [23] is that we consider investment in two risky assets modelled by the CEV where the instantaneous volatilities of the two risky assets form a $2 \times 2$ matrix $n=\left\{n_{p, q}\right\}_{2 \times 2}$ such that $n n^{T}$ is positive definite instead of one risky asset. We used the power transformation, variable change instead of Legendre transformation method and dual theory. 


\section{Materials and Method}

\subsection{Financial Market Model}

Consider a portfolio comprising of one risk free asset and two risky assets in a financial market which is open continuously for an interval $t \in[0, T], T$ the expiration date of the investment. Let $\left\{\mathcal{Z}_{0}(t), \mathcal{Z}_{1}(t), \mathcal{Z}_{2}(t): t \geq 0\right\}$ be standard Brownian motion defined on a complete probability space $(\Omega, F, P)$ where $\Omega$ is a real space and $P$ is a probability measure and $F$ is the filtration which represents the information generated by the three Brownian motions.

Let $\mathcal{S}_{t}(t)$ denote the price of the risk free asset at time $t$ and the model is given as follows

$$
\left\{\begin{array}{l}
\frac{d \mathcal{S}_{0}(t)}{\mathcal{S}_{0}(t)}=\mathcal{R}(t) d t \\
\mathcal{S}_{0}(0)=s_{0}>0
\end{array}\right.
$$

where $\mathcal{R}(t)$ is the interest rate which follows the CIR process and is given by the stochastic differential equation below

$$
\left\{\begin{array}{c}
d \mathcal{R}(t)=(a-b \mathcal{R}(t)) d t-\delta \sqrt{\mathcal{R}(t)} d \mathcal{Z}_{0}(t) \\
\mathcal{R}(0)=\mathcal{R}_{0}>0
\end{array},\right.
$$

where $a, b$, and $\delta$ are positive real numbers such that the following condition holds $\delta^{2}<2 a$ called the Feller's condition [23].

Let $\mathcal{S}_{1}(t)$ and $\mathcal{S}_{2}(t)$ denote the prices of two different stocks which are described by the CEV model and the dynamics of the stock market prices are described by the stochastic differential equations at $t \geq 0$ as follows

$$
\begin{aligned}
& \frac{d \mathcal{S}_{1}(t)}{\mathcal{S}_{1}(t)}=m_{1} d t+n_{11} \mathcal{S}_{1}^{\beta}(t) d \mathcal{Z}_{1}(t)+n_{12} \mathcal{S}_{1}^{\beta}(t) d \mathcal{Z}_{2}(t) \\
& \frac{d \mathcal{S}_{2}(t)}{\mathcal{S}_{2}(t)}=m_{2} d t+n_{21} \mathcal{S}_{2}^{\beta}(t) d \mathcal{Z}_{1}(t)+n_{22} \mathcal{S}_{1}^{\beta}(t) d \mathcal{Z}_{2}(t)
\end{aligned}
$$

where $m_{1}$ and $m_{2}$ are appreciation rate of the two risky assets, $n_{11}, n_{12}, n_{21}, n_{22}$ are instantaneous volatilities and form a $2 \times 2$ matrix $n=\left\{n_{p, q}\right\}_{2 \times 2}$ such that $n n^{T}$ is positive definite and $\beta<0$ represent elasticity parameter, see [26] for details. Note that if $\beta=0$, the stock market price is modelled by GBM.

\section{Optimization Problem}

Let $\varphi$ be the optimal investment plan and we define the utility $\mathcal{K}$ attained by the investor from a given state $z$ at time $t$ as

$$
\left.\mathcal{N}_{\varphi}\left(t, \mathcal{R}, s_{1}, s_{2}, h\right)=E_{\varphi} \mathcal{K}(\mathcal{H}(T)) \mid \mathcal{R}(t)=\mathcal{R}, \mathcal{S}_{1}(t)=s_{1}, \mathcal{S}_{2}(t)=s_{2}, \quad \mathcal{H}(t)=h\right],
$$

where $t$ is the time, $\mathcal{R}$ is the risk free interest rate and $h$ is the wealth. The objective here is to determine the optimal portfolio strategy and the optimal value function of the investor given as

$$
\varphi^{*} \text { and } \mathcal{N}\left(t, \mathcal{R}, s_{1}, s_{2}, h\right)=\sup _{\varphi} \mathcal{N}_{\varphi}\left(t, \mathcal{R}, s_{1}, s_{2}, h\right)
$$

Respectively such that

$$
\mathcal{N}_{\varphi^{*}}\left(t, \mathcal{R}, s_{1}, s_{2}, h\right)=\mathcal{N}\left(t, \mathcal{R}, s_{1}, s_{2}, h\right)
$$

Let $\mathcal{H}(t)$ be the insurer's wealth at time $t$ and then the differential form associated with the fund size is given as:

$$
\mathrm{d} \mathcal{H}(t)=\mathcal{H}(t)\left(\varphi_{0} \frac{d \mathcal{S}_{0}(t)}{\mathcal{S}_{0}(t)}+\varphi_{1} \frac{d \mathcal{S}_{1}(t)}{\mathcal{S}_{1}(t)}+\varphi_{2} \frac{d \mathcal{S}_{2}(t)}{\mathcal{S}_{2}(t)}\right)
$$


substituting (2.1), (2.3) and (2.4) into (3.4), we have

$$
\mathrm{d} \mathcal{H}(t)=\mathcal{H}(t)\left(\begin{array}{c}
\left(\varphi_{1}\left(m_{1}-\mathcal{R}\right)+\varphi_{2}\left(m_{2}-\mathcal{R}\right)+\mathcal{R}\right) d t \\
+\left(\varphi_{1} n_{11} \mathcal{S}_{1}^{\beta}(t)+\varphi_{2} n_{21} \mathcal{S}_{2}^{\beta}(t)\right) d \mathcal{Z}_{1} \\
+\left(\varphi_{1} n_{12} \mathcal{S}_{1}^{\beta}(t)+\varphi_{2} n_{22} \mathcal{S}_{2}^{\beta}(t)\right) d \mathcal{Z}_{2} \\
\mathcal{H}(0)=h_{0}
\end{array}\right)
$$

Where $\varphi_{0}, \varphi_{1}$ and $\varphi_{2}$ are the optimal investment plans for the risk-free asset and the two risky assets respectively, such that $\varphi_{0}=1-\varphi_{1}-\varphi_{2}$.

Applying the Ito's lemma and maximum principle in [25], the Hamilton Jacobi Bellman (HJB) equation which is a nonlinear PDE associated with (3.5) is obtained by maximizing $\mathcal{N}_{\varphi^{*}}\left(t, \mathcal{R}, s_{1}, s_{2}, h\right)$ subject to the insurer's wealth as follows

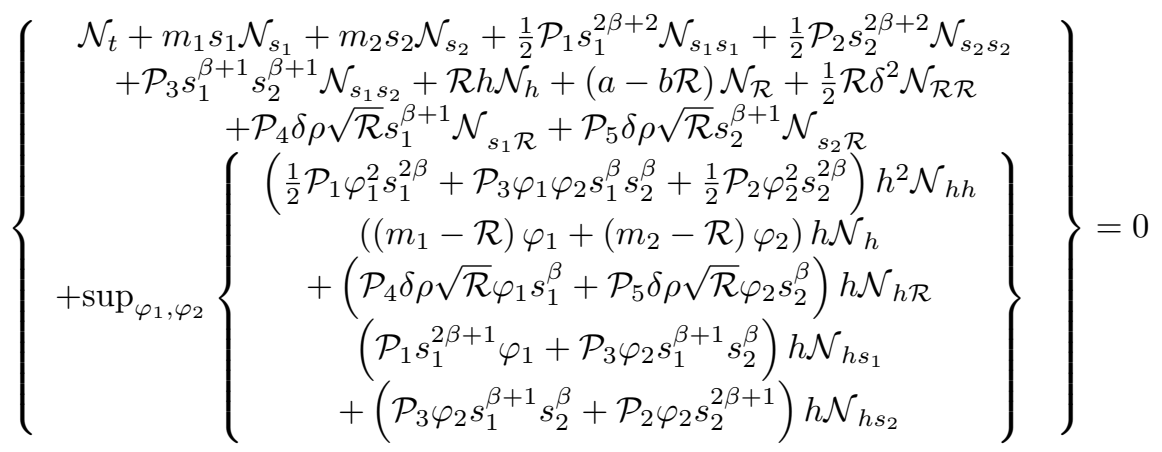

where

$$
\left\{\begin{array}{c}
\mathcal{P}_{1}=n_{11}^{2}+n_{12}^{2}, \quad \mathcal{P}_{2}=n_{21}^{2}+n_{22}^{2}, \mathcal{P}_{3}=n_{11} n_{21}+n_{12} n_{22}, \\
\mathcal{P}_{4}=n_{11}+n_{12}, \mathcal{P}_{5}=n_{21}+n_{22}
\end{array}\right.
$$

Differentiating (3.6) with respect to $\varphi_{1}$ and $\varphi_{2}$, we obtain the first order maximizing condition for equation (3.6) as

$$
\begin{aligned}
& \varphi_{1}^{*}=\frac{\left[\mathcal{P}_{3} s_{1}^{\beta}\left(m_{2}-\mathcal{R}\right)-\mathcal{P}_{2} s_{2}^{\beta}\left(m_{1}-\mathcal{R}\right)\right]}{h\left(\mathcal{P}_{1} \mathcal{P}_{2}-\mathcal{P}_{3}^{2}\right) s_{1}^{2 \beta} s_{2}^{\beta}} \frac{\mathcal{N}_{h}}{\mathcal{N}_{h h}}-s_{1} \frac{\mathcal{N}_{h s_{1}}}{h \mathcal{N}_{h h}}-\frac{\left(\mathcal{P}_{2} \mathcal{P}_{4}-\mathcal{P}_{3} \mathcal{P}_{5}\right) \sqrt{\mathcal{R}} \delta \rho}{h\left(\mathcal{P}_{1} \mathcal{P}_{2}-\mathcal{P}_{3}^{2}\right) s_{1}^{\beta}} \frac{\mathcal{N}_{h \mathcal{R}}}{\mathcal{N}_{h h}} \\
& \varphi_{2}^{*}=\frac{\left[\mathcal{P}_{3} s_{2}^{\beta}\left(m_{1}-\mathcal{R}\right)-\mathcal{P}_{1} s_{1}^{\beta}\left(m_{2}-\mathcal{R}\right)\right]}{h\left(\mathcal{P}_{1} \mathcal{P}_{2}-\mathcal{P}_{3}^{2}\right) s_{1}^{\beta} s_{2}^{2 \beta}} \frac{\mathcal{N}_{h}}{\mathcal{N}_{h h}}-s_{2} \frac{\mathcal{N}_{h s_{2}}}{h \mathcal{N}_{h h}}-\frac{\left(\mathcal{P}_{1} \mathcal{P}_{5}-\mathcal{P}_{3} \mathcal{P}_{4}\right) \sqrt{\mathcal{R}} \delta \rho}{h\left(\mathcal{P}_{1} \mathcal{P}_{2}-\mathcal{P}_{3}^{2}\right) s_{2}^{\beta}} \frac{\mathcal{N}_{h \mathcal{R}}}{\mathcal{N}_{h h}}
\end{aligned}
$$

Substituting (3.7) and (3.8) into (3.6), we have

$$
\left\{\begin{array}{c}
\mathcal{N}_{t}+m_{1} s_{1} \mathcal{N}_{s_{1}}+m_{2} s_{2} \mathcal{N}_{s_{2}}+\mathcal{R} h \mathcal{N}_{h}+\frac{1}{2} \mathcal{P}_{1} s_{1}^{2 \beta+2} \mathcal{N}_{s_{1} s_{1}}+\frac{1}{2} \mathcal{P}_{2} s_{2}^{2 \beta+2} \mathcal{N}_{s_{2} s_{2}} \\
+\mathcal{P}_{3} s_{1}^{\beta+1} s_{2}^{\beta+1} \mathcal{N}_{s_{1} s_{2}}+(a-b \mathcal{R}) \mathcal{N}_{\mathcal{R}}+\frac{1}{2} \mathcal{R} \delta^{2} \mathcal{N}_{\mathcal{R} \mathcal{R}}+\mathcal{P}_{4} \delta \rho \sqrt{\mathcal{R}} s_{1}^{\beta+1} \mathcal{N}_{s_{1} \mathcal{R}} \\
+\mathcal{P}_{5} \delta \rho \sqrt{\mathcal{R}} s_{2}^{\beta+1} \mathcal{N}_{s_{2} \mathcal{R}}+\frac{1}{2}\left(\frac{\mathcal{P}_{6}}{s_{1}^{\beta} s_{2}^{\beta}}-\frac{\mathcal{P}_{7}}{s_{1}^{2 \beta}}-\frac{\mathcal{P}_{8}}{s_{2}^{2 \beta}}\right) \frac{\mathcal{N}_{\mathcal{R}}^{2}}{\mathcal{N}_{h h}}-\left(m_{1}-\mathcal{R}\right) s_{1} \frac{\mathcal{N}_{h} \mathcal{N}_{h s_{1}}}{\mathcal{N}_{h h}} \\
-\left(m_{2}-\mathcal{R}\right) s_{2} \frac{\mathcal{N}_{h} \mathcal{N}_{h s_{2}}}{\mathcal{N}_{h h}}-\delta \rho \sqrt{\mathcal{R}}\left(\frac{\mathcal{P}_{9}\left(m_{1}-\mathcal{R}\right)}{s_{1}^{\beta}}+\frac{\mathcal{P}_{10}\left(m_{2}-\mathcal{R}\right)}{s_{2}^{\beta}}\right) \frac{\mathcal{N}_{h} \mathcal{N}_{h \mathcal{R}}}{\mathcal{N}_{h h}}-\frac{1}{2} \mathcal{P}_{1} s_{1}^{2 \beta+2} \frac{\mathcal{N}_{h s_{1}}^{2}}{\mathcal{N}_{h h}} \\
-\frac{1}{2} \mathcal{P}_{2} s_{2}^{2 \beta+2} \frac{\mathcal{N}_{h s_{2}}^{2}}{\mathcal{N}_{h h}}-\frac{1}{2} \mathcal{P}_{11} \rho^{2} \delta^{2} \mathcal{R} \frac{\mathcal{N}_{h \mathcal{R}}^{2}}{\mathcal{N}_{h h}}-\mathcal{P}_{3} s_{1}^{\beta+1} s_{2}^{\beta+1} \frac{\mathcal{N}_{h s_{1}} \mathcal{N}_{h s_{2}}}{\mathcal{N}_{h h}}
\end{array}\right\}=0
$$

where 


$$
\left\{\begin{array}{c}
\mathcal{P}_{1}=n_{11}^{2}+n_{12}^{2}, \quad \mathcal{P}_{2}=n_{21}^{2}+n_{22}^{2}, \mathcal{P}_{3}=n_{11} n_{21}+n_{12} n_{22}, \\
\mathcal{P}_{4}=n_{11}+n_{12}, \mathcal{P}_{5}=n_{21}+n_{22}, \mathcal{P}_{6}=\frac{2 \mathcal{P}_{3}\left(m_{1}-\mathcal{R}\right)\left(m_{2}-\mathcal{R}\right)}{\left(\mathcal{P}_{1} \mathcal{P}_{2}-\mathcal{P}_{3}^{2}\right)} \\
\mathcal{P}_{7}=\frac{\mathcal{P}_{2}\left(m_{1}-\mathcal{R}\right)^{2}}{\left(\mathcal{P}_{1} \mathcal{P}_{2}-\mathcal{P}_{3}^{2}\right)}, \mathcal{P}_{8}=\frac{\mathcal{P}_{1}\left(m_{2}-\mathcal{R}\right)^{2}}{\left(\mathcal{P}_{1} \mathcal{P}_{2}-\mathcal{P}_{3}^{2}\right)}, \mathcal{P}_{9}=\frac{\left(\mathcal{P}_{2} \mathcal{P}_{4}-\mathcal{P}_{3} \mathcal{P}_{5}\right)}{\left(\mathcal{P}_{1} \mathcal{P}_{2}-\mathcal{P}_{3}^{2}\right)} \\
\mathcal{P}_{10}=\frac{\left(\mathcal{P}_{1} \mathcal{P}_{5}-\mathcal{P}_{3} \mathcal{P}_{4}\right)}{\left(\mathcal{P}_{1} \mathcal{P}_{2}-\mathcal{P}_{3}^{2}\right)}, \mathcal{P}_{11}=\frac{\left(\mathcal{P}_{2} \mathcal{P}_{4}^{2}+\mathcal{P}_{1} \mathcal{P}_{5}^{2}-2 \mathcal{P}_{3} \mathcal{P}_{4} \mathcal{P}_{5}\right)}{\left(\mathcal{P}_{1} \mathcal{P}_{2}-\mathcal{P}_{3}^{2}\right)}
\end{array}\right.
$$

From [27], we assumed that the optimal investment plan for risky assets' prices are known based on the assumption that

$$
m_{1} \varphi_{1}^{*}+m_{2} \varphi_{2}^{*}=\alpha
$$

where $\alpha$ is a constant

Substituting (3.7) and (3.8) into (3.11), we derive an expression for $\frac{1}{s_{1}^{\beta} s_{2}^{\beta}}$ as

$$
\frac{1}{s_{1}^{\beta} s_{2}^{\beta}}=\frac{\mathcal{P}_{1} \mathcal{P}_{2}-\mathcal{P}_{3}^{2}}{\mathcal{P}_{3}\left(2 m_{1} m_{2}-m_{1} \mathcal{R}-m_{2} \mathcal{R}\right)}\left[\begin{array}{c}
\alpha h \frac{\mathcal{L}_{x x}}{\mathcal{L}_{x}}+\frac{\mathcal{P}_{2} m_{1}\left(m_{1}-\mathcal{R}\right)}{\left(\mathcal{P}_{1} \mathcal{P}_{2}-\mathcal{P}_{3}^{2}\right) s_{1}^{2 \beta}}+m_{1} s_{1} \frac{\mathcal{N}_{h s_{1}}}{\mathcal{N}_{h}}+\frac{\left(\mathcal{P}_{2} \mathcal{P}_{4}-\mathcal{P}_{3} \mathcal{P}_{5}\right) m_{1} \sqrt{\mathcal{R}} \delta \rho}{\left(\mathcal{P}_{1} \mathcal{P}_{2}-\mathcal{P}_{3}^{2}\right) s_{1}^{\beta}} \frac{\mathcal{N}_{h \mathcal{R}}}{\mathcal{N}_{h}} \\
+\frac{\mathcal{P}_{1} m_{2}\left(m_{2}-\mathcal{R}\right)}{\left(\mathcal{P}_{1} \mathcal{P}_{2}-\mathcal{P}_{3}^{2}\right) s_{2}^{2 \beta}}+m_{2} s_{2} \frac{\mathcal{N}_{h s_{2}}}{\mathcal{N}_{h}}+\frac{\left(\mathcal{P}_{1} \mathcal{P}_{5}-\mathcal{P}_{3} \mathcal{P}_{4}\right) m_{2} \sqrt{\mathcal{R}} \delta \rho}{\left(\mathcal{P}_{1} \mathcal{P}_{2}-\mathcal{P}_{3}^{2}\right) s_{2}^{\beta}} \frac{\mathcal{N}_{h \mathcal{R}}}{\mathcal{N}_{h}}
\end{array}\right]
$$

Substituting (3.12) into (3.9), we have

$$
\left\{\begin{array}{c}
\mathcal{N}_{t}+m_{1} s_{1} \mathcal{N}_{s_{1}}+m_{2} s_{2} \mathcal{N}_{s_{2}}+\left(\mathcal{R}+\omega_{1}\right) h \mathcal{N}_{h}+\frac{1}{2} \mathcal{P}_{1} s_{1}^{2 \beta+2} \mathcal{N}_{s_{1} s_{1}}+\frac{1}{2} \mathcal{P}_{2} s_{2}^{2 \beta+2} \mathcal{N}_{s_{2} s_{2}} \\
+\mathcal{P}_{3} s_{1}^{\beta+1} s_{2}^{\beta+1} \mathcal{N}_{s_{1} s_{2}}+(a-b \mathcal{R}) \mathcal{N}_{\mathcal{R}}+\frac{1}{2} \mathcal{R} \delta^{2} \mathcal{N}_{\mathcal{R} \mathcal{R}}+\mathcal{P}_{4} \delta \rho \sqrt{\mathcal{R}} s_{1}^{\beta+1} \mathcal{N}_{s_{1} \mathcal{R}} \\
+\mathcal{P}_{5} \delta \rho \sqrt{\mathcal{R}} s_{2}^{\beta+1} \mathcal{N}_{s_{2} \mathcal{R}}+\frac{1}{2}\left(\begin{array}{c}
\omega_{2} s_{1}^{-2 \beta} \\
+\omega_{3} s_{2}^{-2 \beta}
\end{array}\right) \frac{\mathcal{N}_{\mathcal{R}}^{2}}{\mathcal{N}_{h h}}+\left(\omega_{4}-\left(m_{1}-\mathcal{R}\right) s_{1}\right) \frac{\mathcal{N}_{h} \mathcal{N}_{h s_{1}}}{\mathcal{N}_{h h}} \\
+\left(\omega_{5}-\left(m_{2}-\mathcal{R}\right) s_{2}\right) \frac{\mathcal{N}_{h} \mathcal{N}_{h s_{2}}}{\mathcal{N}_{h h}}+\left(\begin{array}{c}
\omega_{6} s_{1}^{-\beta} \\
+\omega_{7} s_{2}^{-\beta}
\end{array}\right) \frac{\mathcal{N}_{h} \mathcal{N}_{h \mathcal{R}}}{\mathcal{N}_{h h}}-\frac{1}{2} \mathcal{P}_{1} s_{1}^{2 \beta+2} \frac{\mathcal{N}_{h s_{1}}^{2}}{\mathcal{N}_{h h}} \\
-\frac{1}{2} \mathcal{P}_{2} s_{2}^{2 \beta+2} \frac{\mathcal{N}_{h s_{2}}^{2}}{\mathcal{N}_{h h}}-\frac{1}{2} \mathcal{P}_{11} \rho^{2} \delta^{2} \mathcal{R} \frac{\mathcal{N}_{h \mathcal{R}}^{2}}{\mathcal{N}_{h h}}-\mathcal{P}_{3} s_{1}^{\beta+1} s_{2}^{\beta+1} \frac{\mathcal{N}_{h s_{1}} \mathcal{N}_{h s_{2}}}{\mathcal{N}_{h h}}
\end{array}\right\}=0
$$

where,

$$
\left\{\begin{array}{c}
\omega_{1}=\frac{\mathcal{P}_{6}\left(\left(\mathcal{P}_{1} \mathcal{P}_{2}-\mathcal{P}_{3}^{2}\right) \alpha\right.}{2 \mathcal{P}_{3}\left(2 m_{1} m_{2}-m_{1} \mathcal{R}-m_{2} \mathcal{R}\right)}, \omega_{2}=\frac{\mathcal{P}_{2} \mathcal{P}_{6} m_{1}\left(m_{1}-\mathcal{R}\right)\left(\mathcal{P}_{1} \mathcal{P}_{2}-\mathcal{P}_{3}^{2}\right)}{\mathcal{P}_{3}\left(2 m_{1} m_{2}-m_{1} \mathcal{R}-m_{2} \mathcal{R}\right)}-\mathcal{P}_{7} \\
\omega_{3}=\frac{\mathcal{P}_{1} \mathcal{P}_{6} m_{2}\left(m_{2}-\mathcal{R}\right)\left(\mathcal{P}_{1} \mathcal{P}_{2}-\mathcal{P}_{3}^{2}\right)}{\mathcal{P}_{3}\left(2 m_{1} m_{2}-m_{1} \mathcal{R}-m_{2} \mathcal{R}\right)}-\mathcal{P}_{8}, \omega_{4}=\frac{\mathcal{P}_{6} m_{1} s_{1}\left(m_{2}-\mathcal{R}\right)\left(\mathcal{P}_{1} \mathcal{P}_{2}-\mathcal{P}_{3}^{2}\right)}{2 \mathcal{P}_{3}\left(2 m_{1} m_{2}-m_{1} \mathcal{R}-m_{2} \mathcal{R}\right)} \\
\omega_{5}=\frac{\mathcal{P}_{6} m_{2} s_{2}\left(m_{2}-\mathcal{R}\right)\left(\mathcal{P}_{1} \mathcal{P}_{2}-\mathcal{P}_{3}^{2}\right)}{2 \mathcal{P}_{3}\left(2 m_{1} m_{2}-m_{1} \mathcal{R}-m_{2} \mathcal{R}\right)}, \omega_{6}=\frac{\left(\mathcal{P}_{2} \mathcal{P}_{4}-\mathcal{P}_{3} \mathcal{P}_{5}\right) \mathcal{P}_{6} m_{1} \delta \rho \sqrt{\mathcal{R}}}{2 \mathcal{P}_{3}\left(2 m_{1} m_{2}-m_{1} \mathcal{R}-m_{2} \mathcal{R}\right)}-\mathcal{P}_{9} \delta \rho \sqrt{\mathcal{R}}\left(m_{1}-\mathcal{R}\right) \\
\omega_{7}=\frac{\left(\mathcal{P}_{1} \mathcal{P}_{5}-\mathcal{P}_{3} \mathcal{P}_{4}\right) \mathcal{P}_{6} m_{1} \delta \rho \sqrt{\mathcal{R}}}{2 \mathcal{P}_{3}\left(2 m_{1} m_{2}-m_{1} \mathcal{R}-m_{2} \mathcal{R}\right)}-\mathcal{P}_{10} \delta \rho \sqrt{\mathcal{R}}\left(m_{2}-\mathcal{R}\right)
\end{array}\right.
$$

Where, $\mathcal{N}\left(t, \mathcal{R}, s_{1}, s_{2}, h\right)=\mathcal{K}(h)$ and $\mathcal{K}(h)$ is the marginal utility of the investor. Next, we proceed to solve (3.13) for $\mathcal{N}$ using exponential utility, after which we substitute the solution into (3.7) and (3.8) for the optimal investment plan using power transformation, variable change proposed by [28] and asymptotic expansion method in [23].

\section{Results \& Conclusion}

\subsection{Optimal Investment Plan for an Investor with CARA Utility}

Consider an investor with exponential utility function which exhibit constant absolute risk aversion (CARA).Here, we choose the exponential utility function similar to the one in [21, 27]. Assume the investor takes an exponential utility given as 


$$
\mathcal{K}(h)=-\frac{1}{\theta} e^{-\theta h}
$$

where $\theta>0$ is the risk aversion coefficient.

From equation (4.1), we construct a solution to (3.13) similar to the one in [21,27] as follows:

$$
\left.\begin{array}{c}
\left\{\begin{array}{c}
\mathcal{N}\left(t, \mathcal{R}, s_{1}, s_{2}, h\right)=-\frac{1}{\theta} e^{-\left(\theta v\left(t, \mathcal{R}, s_{1}\right)+\theta w\left(t, \mathcal{R}, s_{2}\right)+\theta h g(t)\right)} \\
v\left(T, \mathcal{R}, s_{1}\right)=w\left(T, \mathcal{R}, s_{2}\right)=0, g(T)=1
\end{array}\right. \\
\mathcal{N}_{t}=-\theta \mathcal{N}\left(v_{t}+w_{t}+h g_{t}\right), \mathcal{N}_{h}=-\theta \mathcal{N} g, \mathcal{N}_{h h}=\theta^{2} \mathcal{N} g^{2}, \mathcal{N}_{h s_{1}}=\theta^{2} \mathcal{N} g v_{s_{1}} \\
\mathcal{N}_{h s_{2}}=\theta^{2} \mathcal{N} g w_{s_{2}}, \mathcal{N}_{h \mathcal{R}}=\theta^{2} \mathcal{N} g\left(v_{\mathcal{R}}+w_{\mathcal{R}}\right), \mathcal{N}_{s_{1}}=-\theta \mathcal{N} v_{s_{1}}, \mathcal{N}_{s_{2}}=-\theta \mathcal{N} w_{s_{2}} \\
\mathcal{N}_{s_{1} s_{1}}=\mathcal{N}\left(\theta^{2} v_{s_{1}}^{2}-\theta v_{s_{1} s_{1}}\right), \mathcal{N}_{s_{2} s_{2}}=\mathcal{N}\left(\theta^{2} w_{s_{2}}^{2}-\theta w_{s_{2} s_{2}}\right), \mathcal{N}_{s_{1} s_{2}}=\theta^{2} \mathcal{N} g v_{s_{1}} w_{s_{2}} \\
\mathcal{N}_{\mathcal{R}}=-\theta \mathcal{N}\left(v_{\mathcal{R}}+w_{\mathcal{R}}\right), \mathcal{N}_{\mathcal{R} \mathcal{R}}=\mathcal{N}\left(\theta^{2}\left(v_{\mathcal{R}}+w_{\mathcal{R}}\right)^{2}-\theta\left(v_{\mathcal{R} \mathcal{R}}+w_{\mathcal{R} \mathcal{R}}\right)\right) \\
\mathcal{N}_{\mathcal{R} s_{1}}=\mathcal{N}\left(\theta^{2}\left(v_{\mathcal{R}} v_{s_{1}}+w_{\mathcal{R}} v_{s_{1}}\right)-\theta v_{\mathcal{R} s_{1}}\right), \mathcal{N}_{\mathcal{R} s_{2}}=\mathcal{N}\left(\theta^{2}\left(v_{\mathcal{R}} w_{s_{2}}+w_{\mathcal{R}} w_{s_{2}}\right)-\theta w_{\mathcal{R} s_{2}}\right)
\end{array}\right\}
$$

Substituting (4.3) into (3.13), we have

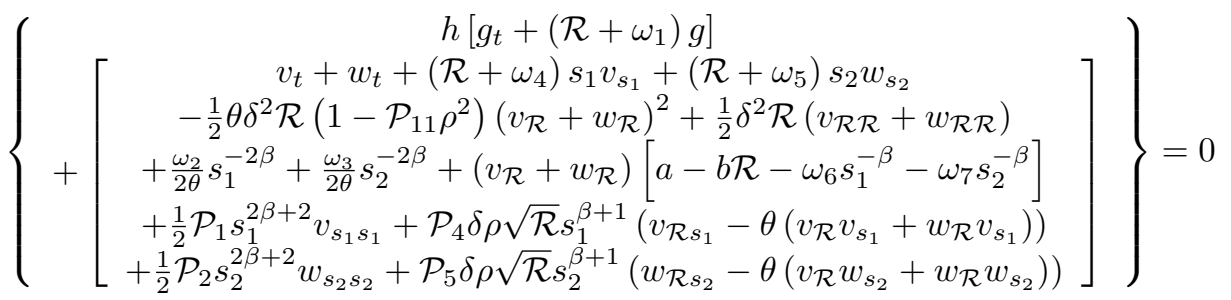

Splitting (4.4) we have

$$
\begin{gathered}
\left\{\begin{array}{c}
g_{t}+\left(\mathcal{R}+\omega_{1}\right) g=0 \\
g(T)=1
\end{array}\right. \\
\left\{\begin{array}{c}
v_{t}+w_{t}+\left(\mathcal{R}+\omega_{4}\right) s_{1} v_{s_{1}}+\left(\mathcal{R}+w_{5}\right) s_{2} w_{s_{2}} \\
-\frac{1}{2} \theta^{2} \mathcal{R}\left(1-\mathcal{P}_{11}^{2}\right)\left(v_{\mathcal{R}}+w_{\mathcal{R}}\right)^{2}+\frac{1}{2}^{2} \mathcal{R}\left(v_{\mathcal{R} \mathcal{R}}+w_{\mathcal{R} \mathcal{R}}\right) \\
+\frac{w_{2}}{2 \theta} s_{1}^{-2 \beta}+\frac{w_{3}}{2 \theta} s_{2}^{-2 \beta}+\left(v_{\mathcal{R}}+w_{\mathcal{R}}\right)\left[a-b \mathcal{R}-w_{6} s_{1}^{-\beta}-w_{7} s_{2}^{-\beta}\right] \\
+\frac{1}{2} \mathcal{P}_{1} s_{1}^{2 \beta+2} v_{s_{1} s_{1}}+\mathcal{P}_{4} \sqrt{\mathcal{R}} s_{1}^{\beta+1}\left(v_{\mathcal{R} s_{1}}-\theta\left(v_{\mathcal{R}} v_{s_{1}}+w_{\mathcal{R}} v_{s_{1}}\right)\right) \\
+\frac{1}{2} \mathcal{P}_{2} s_{2}^{2 \beta+2} w_{s_{2} s_{2}}+\mathcal{P}_{5} \sqrt{\mathcal{R}} s_{2}^{\beta+1}\left(w_{\mathcal{R} s_{2}}-\theta\left(v_{\mathcal{R}} w_{s_{2}}+w_{\mathcal{R}} w_{s_{2}}\right)\right) \\
v\left(T, \mathcal{R}, s_{1}\right)=w\left(T, \mathcal{R}, s_{2}\right)=0
\end{array}\right]=0
\end{gathered}
$$

Solving equation (4.5) for $g$, we obtain

$$
g(t)=e^{\left(\mathcal{R}+w_{1}\right)(T-t)}
$$

Substituting for $w_{1}=\frac{\mathcal{P}_{6}\left(\left(\mathcal{P}_{1} \mathcal{P}_{2}-\mathcal{P}_{3}^{2}\right)\right.}{2 \mathcal{P}_{3}\left(2 m_{1} m_{2}-m_{1} \mathcal{R}-m_{2} \mathcal{R}\right)}$ in $(4.7)$, we have

$$
g(t)=\operatorname{Exp}\left[\mathcal{R}+\frac{\mathcal{P}_{6}\left(\left(\mathcal{P}_{1} \mathcal{P}_{2}-\mathcal{P}_{3}^{2}\right)\right.}{2 \mathcal{P}_{3}\left(2 m_{1} m_{2}-m_{1} \mathcal{R}-m_{2} \mathcal{R}\right)}\right](T-t)
$$

Lemma 4.1. The solution of equation (4.6) is given as

$$
v\left(t, \mathcal{R}, s_{1}\right)+w\left(t, \mathcal{R}, s_{2}\right)=f(t, \mathcal{R}, y, z)=f^{1}(t, \mathcal{R}, y, z)+\sqrt{ } f^{2}(t, \mathcal{R}, y, z)+f^{3}(t, \mathcal{R}, y, z)
$$


where

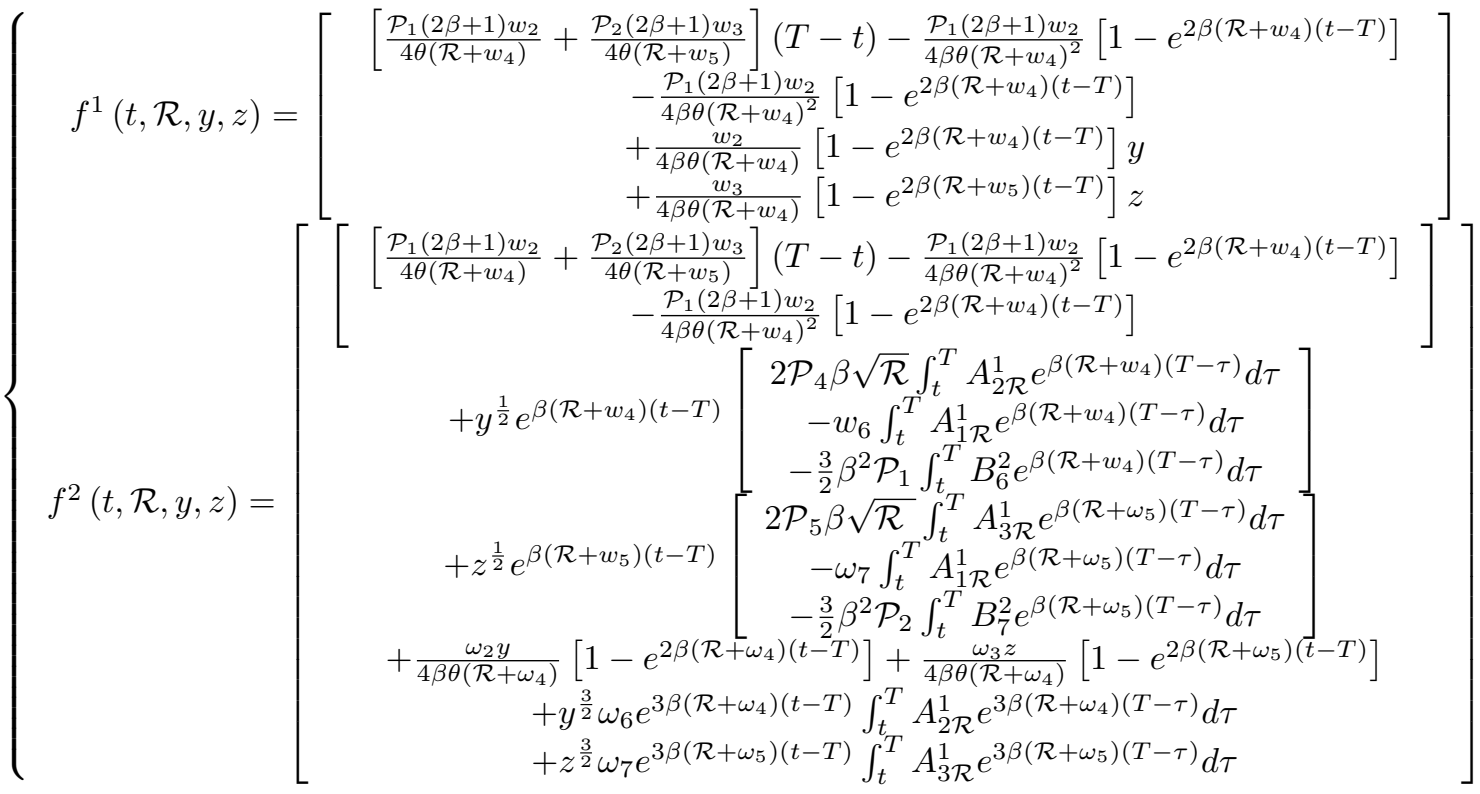

$$
\begin{aligned}
& f^{3}(t, \mathcal{R}, y, z)=\left[\begin{array}{c}
\frac{1}{2} \theta \delta^{2} \mathcal{R}\left(1-\mathcal{P}_{11} \rho^{2}\right) \int_{t}^{T}\left(A_{1 \mathcal{R}}^{1}\right)^{2} d \tau+2 \mathcal{P}_{4} \delta \rho \beta \sqrt{\mathcal{R}} \int_{t}^{T} B_{2 \mathcal{R}}^{2} d \tau \tau \\
+2 \mathcal{P}_{5} \delta \rho \beta \sqrt{\mathcal{R}} \int_{t}^{T} B_{3 \mathcal{R}}^{2} d-\mathcal{P}_{1} \beta(2 \beta+1) \int_{t}^{T} C_{4}^{3} d \tau \\
-\mathcal{P}_{2} \beta(2 \beta+1) \int_{t}^{T} C_{5}^{3} d \tau-(a-b \mathcal{R}) \int_{t}^{T} A_{1 \mathcal{R}}^{1} d \tau-\frac{1}{2} \delta^{2} \mathcal{R} \int_{t}^{T} A_{1 \mathcal{R}}^{1} d \tau
\end{array}\right] \\
& +y^{\frac{1}{2}} e^{\beta\left(\mathcal{R}+\omega_{4}\right)(t-T)}\left[\begin{array}{c}
2 \mathcal{P}_{4} \delta \rho \beta \sqrt{\mathcal{R}} \int_{t}^{T} B_{4 \mathcal{R}}^{2} e^{\beta\left(\mathcal{R}+\omega_{4}\right)(T-\tau)} d \tau \\
+\omega_{6} \int_{t}^{T} B_{1 \mathcal{R}}^{2} e^{\beta\left(\mathcal{R}+\omega_{4}\right)(T-\tau)} d \tau \\
-\frac{3}{2} \beta^{2} \mathcal{P}_{1} \int_{t}^{T} C_{6}^{3} e^{\beta\left(\mathcal{R}+\omega_{4}\right)(T-\tau)} d \tau \\
2 \mathcal{P}_{5} \delta \rho \beta \sqrt{\mathcal{R}} \int_{t}^{T} B_{5 \mathcal{R}}^{2} e^{\beta\left(\mathcal{R}+\omega_{5}\right)(T-\tau)} d \tau \\
+\omega_{7} \int_{t}^{T} B_{1 \mathcal{R}}^{2} e^{\beta\left(\mathcal{R}+\omega_{5}\right)(T-\tau)} d \tau \\
-\frac{3}{2} \beta^{2} \mathcal{P}_{2} \int_{t}^{T} C_{7}^{2} e^{\beta\left(\mathcal{R}+\omega_{5}\right)(T-\tau)} d \tau
\end{array}\right] \\
& {\left[3 \mathcal{P}_{4} \delta \rho \beta \sqrt{\mathcal{R}} \int_{t}^{T} B_{6 \mathcal{R}}^{2} e^{2 \beta\left(\mathcal{R}+\omega_{4}\right)(T-\tau)} d \tau\right.} \\
& +y e^{2 \beta\left(\mathcal{R}+\omega_{4}\right)(t-T)} \quad \begin{array}{c}
-\frac{\omega_{2}}{2 \theta} \int_{t}^{T} e^{2 \beta\left(\mathcal{R}+\omega_{4}\right)(T-\tau)} d \tau-(a-b \mathcal{R}) \int_{t}^{T} A_{2 \mathcal{R}}^{1} e^{2 \beta\left(\mathcal{R}+\omega_{4}\right)(T-\tau)} d \tau \\
+\omega_{6} \int_{t}^{T} B_{2 \mathcal{R}}^{2} e^{2 \beta\left(\mathcal{R}+\omega_{4}\right)(T-\tau)} d \tau-\frac{1}{2} \delta^{2} \mathcal{R} \int_{t}^{T} A_{2 \mathcal{R} \mathcal{R}}^{1} e^{2 \beta\left(\mathcal{R}+\omega_{4}\right)(T-\tau)} d \tau
\end{array} \\
& -2 \mathcal{P}_{1}\left(2 \beta^{2}+\beta(2 \beta+1) \int_{t}^{T} C_{8}^{3} e^{2 \beta\left(\mathcal{R}+\omega_{4}\right)(T-\tau)} d \tau\right. \\
& +\theta \delta^{2} \mathcal{R}\left(1-\mathcal{P}_{11} \rho^{2}\right) \int_{t}^{T} A_{1 \mathcal{R}}^{1} A_{2 \mathcal{R}}^{1} e^{2 \beta\left(\mathcal{R}+\omega_{4}\right)(T-\tau)} d \tau \\
& 3 \mathcal{P}_{5} \delta \rho \beta \sqrt{\mathcal{R}} \int_{t}^{T} B_{7 \mathcal{R}}^{2} e^{2 \beta\left(\mathcal{R}+\omega_{5}\right)(T-\tau)} d \tau \\
& -\frac{\omega_{3}}{2 \theta} \int_{t}^{T} e^{2 \beta\left(\mathcal{R}+\omega_{5}\right)(T-\tau)} d \tau-(a-b \mathcal{R}) \int_{t}^{T} A_{3 \mathcal{R}}^{1} e^{2 \beta\left(\mathcal{R}+\omega_{5}\right)(T-\tau)} d \tau \\
& +z e^{2 \beta\left(\mathcal{R}+\omega_{5}\right)(t-T)} \quad+\omega_{7} \int_{t}^{T} B_{3 \mathcal{R}}^{2} e^{2 \beta\left(\mathcal{R}+\omega_{5}\right)(T-\tau)} d \tau-\frac{1}{2} \delta^{2} \mathcal{R} \int_{t}^{T} A_{3 \mathcal{R} \mathcal{R}}^{1} e^{2 \beta\left(\mathcal{R}+\omega_{5}\right)(T-\tau)} d \tau \\
& -2 \mathcal{P}_{2}\left(2 \beta^{2}+\beta(2 \beta+1) \int_{t}^{T} C_{9}^{3} e^{2 \beta\left(\mathcal{R}+\omega_{5}\right)(T-\tau)} d \tau\right. \\
& +\theta \delta^{2} \mathcal{R}\left(1-\mathcal{P}_{11} \rho^{2}\right) \int_{t}^{T} A_{1 \mathcal{R}}^{1} A_{3 \mathcal{R}}^{1} e^{2 \beta\left(\mathcal{R}+\omega_{5}\right)(T-\tau)} d \tau \\
& +y^{\frac{3}{2}} \omega_{6} e^{3 \beta\left(\mathcal{R}+\omega_{4}\right)(t-T)} \int_{t}^{T} B_{4 \mathcal{R}}^{1} e^{3 \beta\left(\mathcal{R}+\omega_{4}\right)(T-\tau)} d \tau \\
& +z^{\frac{3}{2}} \omega_{7} e^{3 \beta\left(\mathcal{R}+\omega_{5}\right)(t-T)} \int_{t}^{T} B_{5 \mathcal{R}}^{1} e^{3 \beta\left(\mathcal{R}+\omega_{5}\right)(T-\tau)} d \tau \\
& +y^{2} e^{2 \beta\left(\mathcal{R}+\omega_{4}\right)(t-T)}+z^{2} e^{2 \beta\left(\mathcal{R}+\omega_{5}\right)(t-T)}\left[\begin{array}{c}
\frac{1}{2} \theta \delta^{2} \mathcal{R}\left(1-\mathcal{P}_{11} \rho^{2}\right) \int_{t}^{T}\left(A_{2 \mathcal{R}}^{1}\right)^{2} e^{\beta\left(\mathcal{R}+\omega_{4}\right)(T-\tau)} d \tau \\
+\omega_{6} \int_{t}^{T} B_{6 \mathcal{R}}^{2} e^{2 \beta\left(\mathcal{R}+\omega_{4}\right)(T-\tau)} d \tau \\
\frac{1}{2} \theta \delta^{2} \mathcal{R}\left(1-\mathcal{P}_{11} \rho^{2}\right) \int_{t}^{T}\left(A_{3 \mathcal{R}}^{1}\right)^{2} e^{\beta\left(\mathcal{R}+\omega_{4}\right)(T-\tau)} d \tau \\
+\omega_{7} \int_{t}^{T} B_{7 \mathcal{R}}^{2} e^{2 \beta\left(\mathcal{R}+\omega_{4}\right)(T-\tau)} d \tau
\end{array}\right]
\end{aligned}
$$


Proof. Assume

$$
\left\{\begin{array}{c}
v\left(t, \mathcal{R}, s_{1}\right)+w\left(t, \mathcal{R}, s_{2}\right)=f(t, \mathcal{R}, y, z) \\
y=s_{1}^{-2 \beta}, \quad z=s_{2}^{-2 \beta}, f(T, \mathcal{R}, y, z)=0
\end{array}\right.
$$

Then

$$
\left.\begin{array}{rl}
v_{t}+w_{t}=f_{t}, \quad v_{s_{1}}=-2 \beta s_{1}^{-2 \beta-1} f_{y}, \quad v_{s_{1} s_{1}}=2 \beta(2 \beta+1) s_{1}^{-2 \beta-2} f_{y}+4 \beta^{2} s_{1}^{-4 \beta-2} f_{y y} \\
v_{\mathcal{R}}+w_{\mathcal{R}}=f_{\mathcal{R}}, \quad v_{\mathcal{R} R}+w_{\mathcal{R} \mathcal{R}}=f_{\mathcal{R} \mathcal{R}}, v_{\mathcal{R} s_{1}}=-2 \beta s_{1}^{-2 \beta-1} f_{\mathcal{R} y} \\
w_{s_{2}}=-2 \beta s_{2}^{-2 \beta-1} q_{z}, & w_{s_{2} s_{2}}=2 \beta(2 \beta+1) s_{2}^{-2 \beta-2} f_{z}+4 \beta^{2} s_{2}^{-4 \beta-2} f_{z z} \\
& w_{\mathcal{R} s_{2}}=-2 \beta s_{2}^{-2 \beta-1} f_{\mathcal{R} z}
\end{array}\right\}
$$

Substituting (4.10) into (4.6), we have

$$
\left\{\left[\begin{array}{c}
f_{t}-2 \beta y\left(\mathcal{R}+\omega_{4}\right) f_{y}-2 \beta z\left(\mathcal{R}+\omega_{5}\right) f_{z} \\
-\frac{1}{2} \theta \delta^{2} \mathcal{R}\left(1-\mathcal{P}_{11} \rho^{2}\right) f_{\mathcal{R}}^{2}+\frac{1}{2} \delta^{2} \mathcal{R} f_{\mathcal{R} \mathcal{R}} \\
+\frac{\omega_{2}}{2 \theta} y+\frac{\omega_{3}}{2 \theta} z+f_{\mathcal{R}}\left[a-b \mathcal{R}-\omega_{6} \sqrt{y}-\omega_{7} \sqrt{z}\right] \\
+\mathcal{P}_{1} \beta(2 \beta+1) f_{y}+2 \mathcal{P}_{1} \beta^{2} y f_{y y}-2 \mathcal{P}_{4} \delta \rho \beta \sqrt{\mathcal{R}} \sqrt{y}\left(f_{\mathcal{R} y}-\theta f_{\mathcal{R}} f_{y}\right) \\
+\mathcal{P}_{2} \beta(2 \beta+1) f_{z}+2 \mathcal{P}_{2} \beta^{2} z f_{z z}-2 \mathcal{P}_{5} \delta \rho \sqrt{\mathcal{R}} \sqrt{z}\left(f_{\mathcal{R} z}-\theta f_{\mathcal{R}} f_{z}\right)
\end{array}\right]=0\right.
$$

We can rewrite (4.11) as

$$
(E+F+G) f-\frac{1}{2} \theta \delta^{2} \mathcal{R}\left(1-\mathcal{P}_{11} \rho^{2}\right) f_{\mathcal{R}}^{2}=0
$$

Where

$$
\begin{gathered}
E=\left[(a-b \mathcal{R}) f_{\mathcal{R}}+\frac{1}{2} \delta^{2} \mathcal{R} f_{\mathcal{R} \mathcal{R}}\right] \\
F=\left[\begin{array}{c}
f_{t}+\beta\left(\mathcal{P}_{1}(2 \beta+1)-2 y\left(\mathcal{R}+\omega_{4}\right)\right) f_{y}+\frac{\omega_{2}}{2 \theta} y+\frac{\omega_{3}}{2 \theta} z \\
+\beta\left(\mathcal{P}_{2}(2 \beta+1)-2 z\left(\mathcal{R}+\omega_{5}\right)\right) f_{z}+2 \beta^{2} \mathcal{P}_{1} y f_{y y}+2 \beta^{2} \mathcal{P}_{2} z f_{z z}
\end{array}\right] \\
G=\left[\begin{array}{c}
2 \mathcal{P}_{4} \delta \rho \beta \sqrt{\mathcal{R}}\left(\theta f_{\mathcal{R}} f_{y}-f_{\mathcal{R} y}\right) \sqrt{y}+2 \mathcal{P}_{5} \delta \rho \beta \sqrt{\mathcal{R}}\left(\theta f_{\mathcal{R}} f_{z}-f_{\mathcal{R} z}\right) \sqrt{z} \\
-\left(\omega_{6} \sqrt{y}+\omega_{7} \sqrt{z}\right) f_{\mathcal{R}}
\end{array}\right]
\end{gathered}
$$

Next we follow the approach in [11] by applying the asymptotic expansion method to solve the problem in (4.12).

Assume that the volatility follows a slow fluctuating process, we attempt to find an asymptotic solution of (4.12) by a following slow-fluctuating process $r_{\alpha}$ to replace (2.2), in which $0<\varepsilon \ll 1$ is a small positive parameter:

$$
d \mathcal{R}_{\varepsilon}(t)=\left(a-b \mathcal{R}_{\varepsilon}(t)\right) d t-\delta \sqrt{\mathcal{R}_{\varepsilon}(t)} d \mathcal{Z}_{0}(t),
$$

Substituting (4.16) into (4.12) and also replacing $a-b \mathcal{R}(t)$ by $\varepsilon(a-b \mathcal{R}(t))$ and $\sqrt{\mathcal{R}}$ by $\sqrt{\varepsilon} \sqrt{\mathcal{R}}$, we will have

$$
(\varepsilon E+F+\sqrt{\varepsilon} G) f_{\varepsilon}=0
$$

Next, we conjecture a solution for (4.17) as follows

$$
f_{\varepsilon}(t, \mathcal{R}, y, z)=f^{1}(t, \mathcal{R}, y, z)+\sqrt{\varepsilon} f^{2}(t, \mathcal{R}, y, z)+\varepsilon f^{3}(t, \mathcal{R}, y, z)
$$

Substituting (4.18) into (4.14) and simplifying it, we have

$$
\left(\begin{array}{c}
F f^{1}(t, \mathcal{R}, y, z)+\left[F f^{2}(t, \mathcal{R}, y, z)+G f^{1}(t, \mathcal{R}, y, z)\right] \sqrt{\varepsilon} \\
+\left[\begin{array}{c}
E f^{1}(t, \mathcal{R}, y, z)+F f^{3}(t, \mathcal{R}, y, z) \\
+G f^{2}(t, \mathcal{R}, y, z)-\frac{1}{2} \theta \delta^{2} \mathcal{R}\left(1-\mathcal{P}_{11} \rho^{2}\right)\left(f_{\mathcal{R}}^{1}\right)^{2}
\end{array}\right] \varepsilon
\end{array}\right)=0
$$

This implies that

$$
\left\{\begin{array}{c}
F f^{1}(t, \mathcal{R}, y, z)=F\left(p^{1}(t, \mathcal{R}, y)+q^{1}(t, \mathcal{R}, z)\right)=0 \\
f^{1}(T, \mathcal{R}, y, z)=0
\end{array}\right.
$$




$$
\begin{gathered}
\left\{\begin{array}{c}
F f_{2}(t, \mathcal{R}, y, z)+G f_{1}(t, \mathcal{R}, y, z)=0 \\
f^{1}(T, \mathcal{R}, y, z)=f^{2}(T, \mathcal{R}, y, z)=0
\end{array}\right. \\
\left\{\begin{array}{c}
E f^{1}(t, \mathcal{R}, y, z)+F f^{3}(t, \mathcal{R}, y, z)+G f^{2}(t, \mathcal{R}, y, z)=0 \\
f^{1}(T, \mathcal{R}, y, z)=f^{2}(T, \mathcal{R}, y, z)=f^{3}(T, \mathcal{R}, y, z)=0
\end{array}\right.
\end{gathered}
$$

From (4.13), (4.14) and (4.15), equation (4.20), (4.21) and (4.22) can be expressed as

$$
\begin{aligned}
& \left\{\begin{array}{c}
{\left[\begin{array}{c}
f_{t}^{1}+\beta\left(\mathcal{P}_{1}(2 \beta+1)-2 y\left(\mathcal{R}+\omega_{4}\right)\right) f_{y}^{1}+\frac{\omega_{2}}{2 \theta} y+\frac{\omega_{3}}{2 \theta} z \\
+\beta\left(\mathcal{P}_{2}(2 \beta+1)-2 z\left(\mathcal{R}+\omega_{5}\right)\right) f_{z}^{1}+2 \beta^{2} \mathcal{P}_{1} f_{y y}^{1} y+2 \beta^{2} \mathcal{P}_{2} f_{z z}^{1} z
\end{array}\right]=0} \\
f^{1}(T, \mathcal{R}, y, z)=0
\end{array}\right. \\
& \left\{\begin{array}{c}
f_{t}^{2}+\beta\left(\mathcal{P}_{1}(2 \beta+1)-2 y\left(\mathcal{R}+\omega_{4}\right)\right) f_{y}^{2}+\frac{\omega_{2}}{2 \theta} y+\frac{\omega_{3}}{2 \theta} z \\
+\beta\left(\mathcal{P}_{2}(2 \beta+1)-2 z\left(\mathcal{R}+\omega_{5}\right)\right) f_{z}^{2}+2 \beta^{2} \mathcal{P}_{1} f_{y y}^{2} y+2 \beta^{2} \mathcal{P}_{2} f_{z z}^{2} z \\
+2 \mathcal{P}_{4} \delta \rho \beta \sqrt{\mathcal{R}}\left(\theta f_{\mathcal{R}}^{1} f_{y}^{1}-f_{y \mathcal{R}}^{1}\right) \sqrt{y}-\left(\omega_{6} \sqrt{y}+\omega_{7} \sqrt{z}\right) f_{\mathcal{R}}^{1} \\
+2 \mathcal{P}_{5} \delta \rho \beta \sqrt{\mathcal{R}}\left(\theta f_{\mathcal{R}}^{1} f_{z}^{1}-f_{\mathcal{R} z}^{1}\right) \sqrt{z} \\
f^{1}(T, \mathcal{R}, y, z)=f^{2}(T, \mathcal{R}, y, z)=0
\end{array}\right]=0 \\
& \left\{\left[\begin{array}{c}
f_{t}^{3}+\beta\left(\mathcal{P}_{1}(2 \beta+1)-2 y\left(\mathcal{R}+\omega_{4}\right)\right) f_{y}^{3}+\frac{\omega_{2}}{2 \theta} y+\frac{\omega_{3}}{2 \theta} z \\
+\beta\left(\mathcal{P}_{2}(2 \beta+1)-2 z\left(\mathcal{R}+\omega_{5}\right)\right) f_{z}^{3}+2 \beta^{2} \mathcal{P}_{1} f_{y y}^{3} y+2 \beta^{2} \mathcal{P}_{2} f_{z z}^{3} z \\
+2 \mathcal{P}_{4} \delta \rho \sqrt{\mathcal{R}}\left(\theta f_{\mathcal{R}}^{2} f_{y}^{2}-f_{y \mathcal{R}}^{2}\right) \sqrt{y}-\left(\omega_{6} \sqrt{y}+\omega_{7} \sqrt{z}\right) f_{\mathcal{R}}^{2} \\
+2 \mathcal{P}_{5} \delta \rho \beta \sqrt{\mathcal{R}}\left(\theta f_{\mathcal{R}}^{2} f_{z}^{2}-f_{\mathcal{R} z}^{2}\right) \sqrt{z}-\frac{1}{2} \theta \delta^{2} \mathcal{R}\left(1-\mathcal{P}_{11} \rho^{2}\right)\left(f_{\mathcal{R}}^{1}\right)^{2} \\
(a-b \mathcal{R}) f_{\mathcal{R}}^{1}+\frac{1}{2} \delta^{2} \mathcal{R} f_{\mathcal{R} \mathcal{R}}^{1} \\
f^{1}(T, \mathcal{R}, y, z)=f^{2}(T, \mathcal{R}, y, z)=f^{3}(T, \mathcal{R}, y, z)=0
\end{array}\right]=0\right.
\end{aligned}
$$

Next, we move on to solve equation (4.23), (4.24) and (4.25) for $f^{1}, f^{2}$ and $f^{3}$

From (4.23), we conjecture a solution of the form

$$
\left\{\begin{array}{c}
f^{1}(t, \mathcal{R}, y, z)=A_{1}^{1}(t, \mathcal{R})+y A_{2}^{1}(t, \mathcal{R})+z A_{3}^{1}(t, \mathcal{R}) \\
A_{1}^{1}(T, \mathcal{R})=A_{2}^{1}(T, \mathcal{R})=A_{3}^{1}(T, \mathcal{R})=0
\end{array}\right.
$$

and

$$
\left.f_{t}^{1}=A_{1 t}^{1}+y A_{2 t}^{1}+z A_{3 t}^{1}, \quad f_{y}^{1}=A_{2}^{1}, f_{y y}^{1}=0, f_{z}^{1}=A_{3}^{1}, f_{z z}^{1}=0\right\}
$$

Substituting (4.27) in (4.23), we have

$$
\begin{gathered}
\left\{\begin{array}{c}
A_{1 t}^{1}+\mathcal{P}_{1} \beta(2 \beta+1) A_{2}^{1}+\mathcal{P}_{2} \beta(2 \beta+1) A_{3}^{1}=0 \\
A_{1}^{1}(T, \mathcal{R})=A_{2}^{1}(T, \mathcal{R})=A_{3}^{1}(T, \mathcal{R})=0
\end{array}\right. \\
\left\{\begin{array}{c}
A_{2 t}^{1}-2 \beta\left(\mathcal{R}+\omega_{4}\right) A_{2}^{1}+\frac{\omega_{2}}{2 \theta}=0 \\
A_{2}^{1}(T, \mathcal{R})=0
\end{array}\right. \\
\left\{\begin{array}{c}
A_{3 t}^{1}-2 \beta\left(\mathcal{R}+\omega_{5}\right) A_{3}^{1}+\frac{\omega_{3}}{2 \theta}=0 \\
A_{3}^{1}(T, \mathcal{R})=0
\end{array}\right.
\end{gathered}
$$

Solving (4.28), (4.29) and (4.30), we have

$$
A_{1}^{1}(t, \mathcal{R})=\left[\begin{array}{c}
{\left[\frac{\mathcal{P}_{1}(2 \beta+1) \omega_{2}}{4 \theta\left(\mathcal{R}+\omega_{4}\right)}+\frac{\mathcal{P}_{2}(2 \beta+1) \omega_{3}}{4 \theta\left(\mathcal{R}+\omega_{5}\right)}\right](T-t)-\frac{\mathcal{P}_{1}(2 \beta+1) \omega_{2}}{4 \beta \theta\left(\mathcal{R}+\omega_{4}\right)^{2}}\left[1-e^{2 \beta\left(\mathcal{R}+\omega_{4}\right)(t-T)}\right]} \\
-\frac{\mathcal{P}_{1}(2 \beta+1) \omega_{2}}{4 \beta \theta\left(\mathcal{R}+\omega_{4}\right)^{2}}\left[1-e^{2 \beta\left(\mathcal{R}+\omega_{4}\right)(t-T)}\right]
\end{array}\right]
$$


Hence from (4.26)

$$
f^{1}(t, \mathcal{R}, y, z)=\left[\begin{array}{c}
{\left[\frac{\mathcal{P}_{1}(2 \beta+1) \omega_{2}}{4 \theta\left(\mathcal{R}+\omega_{4}\right)}+\frac{\mathcal{P}_{2}(2 \beta+1) \omega_{3}}{4 \theta\left(\mathcal{R}+\omega_{5}\right)}\right](T-t)-\frac{\mathcal{P}_{1}(2 \beta+1) \omega_{2}}{4 \beta \theta\left(\mathcal{R}+\omega_{4}\right)^{2}}\left[1-e^{2 \beta\left(\mathcal{R}+\omega_{4}\right)(t-T)}\right]} \\
-\frac{\mathcal{P}_{1}(2 \beta+1) \omega_{2}}{4 \beta \theta\left(\mathcal{R}+\omega_{4}\right)^{2}}\left[1-e^{2 \beta\left(\mathcal{R}+\omega_{4}\right)(t-T)}\right] \\
+\frac{\omega_{2}}{4 \beta \theta\left(\mathcal{R}+\omega_{4}\right)}\left[1-e^{2 \beta\left(\mathcal{R}+\omega_{4}\right)(t-T)}\right] y \\
+\frac{\omega_{3}}{4 \beta \theta\left(\mathcal{R}+\omega_{4}\right)}\left[1-e^{2 \beta\left(\mathcal{R}+\omega_{5}\right)(t-T)}\right] z
\end{array}\right.
$$

Next, we proceed to solve (4.24), by assuming a solution of the form

$$
f^{2}(t, \mathcal{R}, y, z)=\left[\begin{array}{c}
B_{1}^{2}(t, \mathcal{R})+y^{\frac{1}{2}} B_{2}^{2}(t, \mathcal{R})+z^{\frac{1}{2}} B_{3}^{2}(t, \mathcal{R})+y B_{4}^{2}(t, \mathcal{R}) \\
+z B_{5}^{2}(t, \mathcal{R})+y^{\frac{3}{2}} B_{6}^{2}(t, \mathcal{R})+z^{\frac{3}{2}} B_{7}^{2}(t, \mathcal{R}) \\
B_{1}^{2}(T, \mathcal{R})=B_{2}^{2}(T, \mathcal{R})=B_{3}^{2}(T, \mathcal{R})=B_{4}^{2}(T, \mathcal{R}) \\
=B_{5}^{2}(T, \mathcal{R})=B_{6}^{2}(T, \mathcal{R})=B_{7}^{2}(T, \mathcal{R})=0
\end{array}\right]
$$

and

$$
\left.\begin{array}{c}
f_{t}^{2}=B_{1 t}^{2}+y^{\frac{1}{2}} B_{2 t}^{2}+z^{\frac{1}{2}} B_{3 t}^{2}+y B_{4 t}^{2}+z B_{5 t}^{2}+y^{\frac{3}{2}} B_{6 t}^{2}+z^{\frac{3}{2}} B_{7 t}^{2}, \\
f_{y}^{2}=\frac{1}{2} y^{-\frac{1}{2}} B_{2}^{2}+B_{4}^{2}+\frac{3}{2} y^{\frac{1}{2}} B_{6}^{2}, f_{y y}^{2}=-\frac{1}{4} y^{-\frac{3}{2}} B_{2}^{2}+\frac{3}{4} y^{-\frac{1}{2}} B_{6}^{2}, \\
f_{z}^{2}=\frac{1}{2} z^{-\frac{1}{2}} B_{3}^{2}+B_{5}^{2}+\frac{3}{2} z^{\frac{1}{2}} B_{7}^{2}, f_{z z}^{2}=-\frac{1}{4} z^{-\frac{3}{2}} B_{3}^{2}+\frac{3}{4} z^{-\frac{1}{2}} B_{7}^{2}
\end{array}\right\}
$$

Substituting (4.36) into (4.24), we have

$$
\begin{gathered}
\left\{\begin{array}{c}
B_{1 t}^{2}+\mathcal{P}_{1} \beta(2 \beta+1) B_{4}^{2}+\mathcal{P}_{2} \beta(2 \beta+1) B_{5}^{2}=0 \\
B_{1}^{2}(T, \mathcal{R})=B_{2}^{2}(T, \mathcal{R})=B_{3}^{2}(T, \mathcal{R})=0
\end{array}\right. \\
\left\{\begin{array}{c}
B_{2 t}^{2}-\beta\left(\mathcal{R}+\omega_{4}\right) B_{2}^{2}+\frac{3}{2} \beta^{2} \mathcal{P}_{1} B_{6}^{2}-2 \mathcal{P}_{4} \delta \rho \beta \sqrt{\mathcal{R}} A_{2 \mathcal{R}}^{1}-\omega_{6} A_{1 \mathcal{R}}^{1}=0 \\
B_{2}^{2}(T, \mathcal{R})=0
\end{array}\right. \\
\left\{\begin{array}{c}
B_{3 t}^{2}-\beta\left(\mathcal{R}+\omega_{5}\right) B_{3}^{2}+\frac{3}{2} \beta^{2} \mathcal{P}_{2} B_{7}^{2}-2 \mathcal{P}_{5} \delta \rho \beta \sqrt{\mathcal{R}} A_{3 \mathcal{R}}^{1}-\omega_{7} A_{1 \mathcal{R}}^{1}=0 \\
B_{3}^{2}(T, \mathcal{R})=0
\end{array}\right. \\
\left\{\begin{array}{c}
B_{4 t}^{2}-2 \beta\left(\mathcal{R}+\omega_{4}\right) B_{4}^{2}+\frac{\omega_{2}}{2 \theta}=0 \\
B_{4}^{2}(T, \mathcal{R})=0
\end{array},\right. \\
\left\{\begin{array}{c}
B_{5 t}^{2}-2 \beta\left(\mathcal{R}+\omega_{5}\right) B_{5}^{2}+\frac{\omega_{3}}{2 \theta}=0 \\
B_{5}^{2}(T, \mathcal{R})=0
\end{array}\right. \\
\left\{\begin{array}{c}
B_{6 t}^{2}-3 \beta\left(\mathcal{R}+\omega_{4}\right) B_{6}^{2}+\omega_{6} A_{2 \mathcal{R}}^{1}=0 \\
B_{6}^{2}(T, \mathcal{R})=0
\end{array}\right. \\
\left\{\begin{array}{c}
B_{7 t}^{2}-3 \beta\left(\mathcal{R}+\omega_{5}\right) B_{7}^{2}+\omega_{7} A_{3 \mathcal{R}}^{1}=0 \\
B_{7}^{2}(T, \mathcal{R})=0
\end{array}\right.
\end{gathered}
$$

Solving equation (4.37) - (4.43), we have

$$
\begin{aligned}
& B_{1}^{2}(t, \mathcal{R})=\left[\begin{array}{c}
{\left[\frac{\mathcal{P}_{1}(2 \beta+1) \omega_{2}}{4 \theta\left(\mathcal{R}+\omega_{4}\right)}+\frac{\mathcal{P}_{2}(2 \beta+1) \omega_{3}}{4 \theta\left(\mathcal{R}+\omega_{5}\right)}\right](T-t)-\frac{\mathcal{P}_{1}(2 \beta+1) \omega_{2}}{4 \beta \theta\left(\mathcal{R}+\omega_{4}\right)^{2}}\left[1-e^{2 \beta\left(\mathcal{R}+\omega_{4}\right)(t-T)}\right]} \\
-\frac{\mathcal{P}_{1}(2 \beta+1) \omega_{2}}{4 \beta \theta\left(\mathcal{R}+\omega_{4}\right)^{2}}\left[1-e^{2 \beta\left(\mathcal{R}+\omega_{4}\right)(t-T)}\right]
\end{array}\right] \\
& B_{2}^{2}(t, \mathcal{R})=e^{\beta\left(\mathcal{R}+\omega_{4}\right)(t-T)}\left[\begin{array}{c}
2 \mathcal{P}_{4} \delta \rho \beta \sqrt{\mathcal{R}} \int_{t}^{T} A_{2 \mathcal{R}}^{1} e^{\beta\left(\mathcal{R}+\omega_{4}\right)(T-\tau)} d \tau \\
-\omega_{6} \int_{t}^{T} A_{1 \mathcal{R}}^{1} e^{\beta\left(\mathcal{R}+\omega_{4}\right)(T-\tau)} d \tau \\
-\frac{3}{2} \beta^{2} \mathcal{P}_{1} \int_{t}^{T} B_{6}^{2} e^{\beta\left(\mathcal{R}+\omega_{4}\right)(T-\tau)} d \tau \\
2 \mathcal{P}_{5} \delta \rho \beta \sqrt{\mathcal{R}} \int_{t}^{T} A_{3 \mathcal{R}}^{1} e^{\beta\left(\mathcal{R}+\omega_{5}\right)(T-\tau)} d \tau \\
-\omega_{7} \int_{t}^{T} A_{1 \mathcal{R}}^{1} e^{\beta\left(\mathcal{R}+\omega_{5}\right)(T-\tau)} d \tau \\
-\frac{3}{2} \beta^{2} \mathcal{P}_{2} \int_{t}^{T} B_{7}^{2} e^{\beta\left(\mathcal{R}+\omega_{5}\right)(T-\tau)} d \tau
\end{array}\right] \\
& B_{4}^{2}(t, \mathcal{R})=\frac{\omega_{2}}{4 \beta \theta\left(\mathcal{R}+\omega_{4}\right)}\left[1-e^{2 \beta\left(\mathcal{R}+\omega_{4}\right)(t-T)}\right] \\
& B_{5}^{2}(t, \mathcal{R})=\frac{\left.\omega_{3} \omega_{4}\right)}{4 \beta \theta\left(\mathcal{R}+\omega_{4}\right)}\left[1-e^{2 \beta\left(\mathcal{R}+\omega_{5}\right)(t-T)}\right] \\
& B_{6}^{2}(t, \mathcal{R})=\omega_{6} e^{3 \beta\left(\mathcal{R}+\omega_{4}\right)(t-T)} \int_{t}^{T} A_{2 \mathcal{R}}^{1} e^{3 \beta\left(\mathcal{R}+\omega_{4}\right)(T-\tau)} d \tau \\
& B_{7}^{2}(t, \mathcal{R})=\omega_{7} e^{3 \beta\left(\mathcal{R}+\omega_{5}\right)(t-T)} \int_{t}^{T} A_{3 \mathcal{R}}^{1} e^{3 \beta\left(\mathcal{R}+\omega_{5}\right)(T-\tau)} d \tau
\end{aligned}
$$


Substituting (4.44) into (4.35), we have

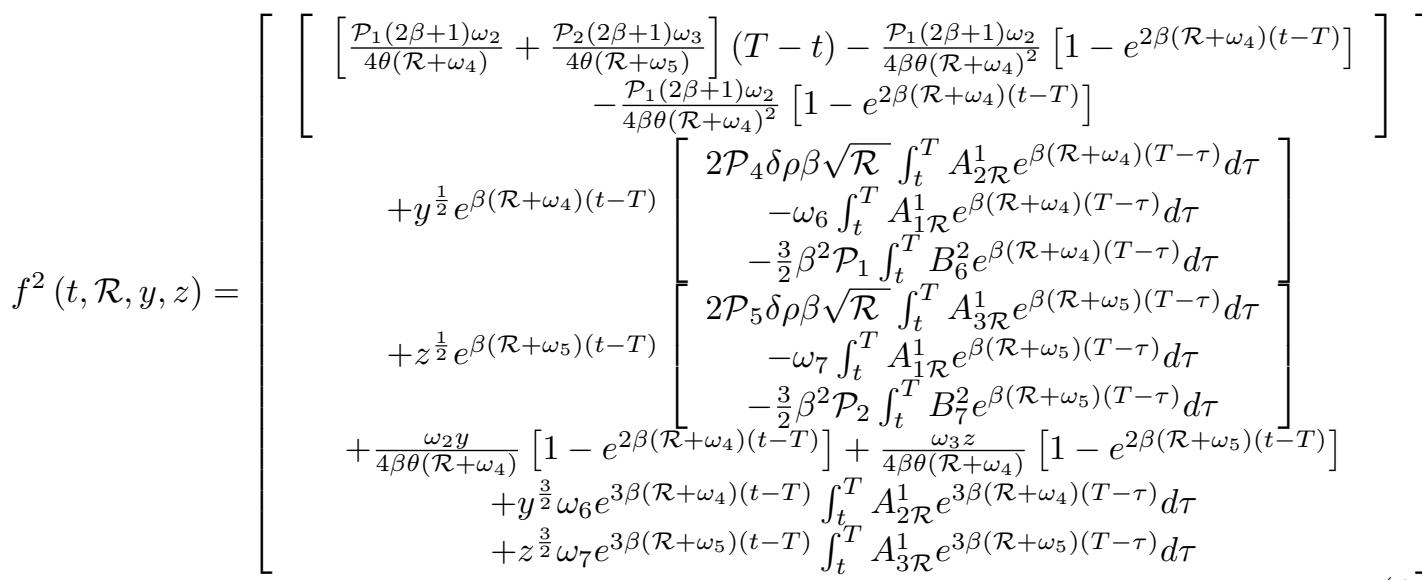

Next, we attempt to solve (4.25), by assuming a solution of the form

$$
f^{3}(t, \mathcal{R}, y, z)=\left[\begin{array}{c}
C_{1}^{3}(t, \mathcal{R})+y^{\frac{1}{2}} C_{2}^{3}(t, \mathcal{R})+z^{\frac{1}{2}} C_{3}^{3}(t, \mathcal{R})+y C_{4}^{3}(t, \mathcal{R})+z C_{5}^{3}(t, \mathcal{R}) \\
+y^{\frac{3}{2}} C_{6}^{3}(t, \mathcal{R})+z^{\frac{3}{2}} C_{7}^{3}(t, \mathcal{R})+y^{2} C_{8}^{3}(t, \mathcal{R})+z^{2} C_{9}^{3}(t, \mathcal{R}) \\
C_{1}^{3}(T, \mathcal{R})=C_{2}^{3}(T, \mathcal{R})=C_{3}^{3}(T, \mathcal{R})=C_{4}^{3}(T, \mathcal{R})=C_{5}^{3}(T, \mathcal{R}) \\
=C_{6}^{3}(T, \mathcal{R})=C_{7}^{3}(T, \mathcal{R})=C_{8}^{3}(T, \mathcal{R})=C_{9}^{3}(T, \mathcal{R})=0
\end{array}\right]
$$

and

$$
\begin{gathered}
f_{t}^{3}=C_{1 t}^{3}+y^{\frac{1}{2}} C_{2 t}^{3}+z^{\frac{1}{2}} C_{3 t}^{3}+y C_{4 t}^{3}+z C_{5 t}^{2}+y^{\frac{3}{2}} C_{6 t}^{3}+z^{\frac{3}{2}} C_{7 t}^{3}+y^{2} C_{8 t}^{3}+z^{2} C_{9 t}^{3}, \\
f_{y}^{3}=\frac{1}{2} y^{-\frac{1}{2}} C_{2}^{3}+C_{4}^{3}+\frac{3}{2} y^{\frac{1}{2}} C_{6}^{3}+2 y C_{8}^{3}, f_{y y}^{3}=-\frac{1}{4} y^{-\frac{3}{2}} C_{2}^{3}+\frac{3}{4} y^{-\frac{1}{2}} C_{6}^{3}+2 C_{8}^{3} \\
f_{z}^{3}=\frac{1}{2} z^{-\frac{1}{2}} C_{3}^{2}+C_{5}^{2}+\frac{3}{2} z^{\frac{1}{2}} C_{7}^{2}+2 z C_{9}^{3}, f_{z z}^{3}=-\frac{1}{4} z^{-\frac{3}{2}} C_{3}^{3}+\frac{3}{4} z^{-\frac{1}{2}} C_{7}^{3}+2 C_{9}^{3} \\
f_{\mathcal{R}}^{1}=A_{1 \mathcal{R}}^{1}+y A_{2 \mathcal{R}}^{1}+z A_{3 \mathcal{R}}^{1}, f_{\mathcal{R} \mathcal{R}}^{1}=A_{1 \mathcal{R} \mathcal{R}}^{1}+y A_{2 \mathcal{R} \mathcal{R}}^{1}+z A_{3 \mathcal{R} \mathcal{R}}^{1} \\
f_{\mathcal{R}}^{2}=B_{1 \mathcal{R}}^{2}+y^{\frac{1}{2}} B_{2 \mathcal{R}}^{2}+z^{\frac{1}{2}} B_{3 \mathcal{R}}^{2}+y B_{4 \mathcal{R}}^{2}+z B_{5 \mathcal{R}}^{2}+y^{\frac{3}{2}} B_{6 \mathcal{R}}^{2}+z^{\frac{3}{2}} B_{7 \mathcal{R}}^{2} \\
f_{y \mathcal{R}}^{2}=\frac{1}{2} y^{-\frac{1}{2}} B_{2 \mathcal{R}}^{2}+B_{4 \mathcal{R}}^{2}+\frac{3}{2} y^{\frac{1}{2}} B_{6 \mathcal{R}}^{2}, f_{z \mathcal{R}}^{2}=\frac{1}{2} z^{-\frac{1}{2}} B_{3 \mathcal{R}}^{2}+B_{5 \mathcal{R}}^{2}+\frac{3}{2} z^{\frac{1}{2}} B_{7 \mathcal{R}}^{2}
\end{gathered}
$$

Substituting (4.47) into (4.25), we have

$$
\begin{aligned}
& \left\{\begin{array}{c}
C_{1 t}^{3}+\mathcal{P}_{1} \beta(2 \beta+1) C_{4}^{3}+\mathcal{P}_{2} \beta(2 \beta+1) C_{5}^{3}+(a-b \mathcal{R}) A_{1 \mathcal{R}}^{1}+\frac{1}{2} \delta^{2} \mathcal{R} A_{1 \mathcal{R} \mathcal{R}}^{1} \\
-\frac{1}{2} \theta \delta^{2} \mathcal{R}\left(1-\mathcal{P}_{11} \rho^{2}\right)\left(A_{1 \mathcal{R}}^{1}\right)^{2}-2 \mathcal{P}_{4} \delta \rho \beta \sqrt{\mathcal{R}} B_{2 \mathcal{R}}^{2}-2 \mathcal{P}_{5} \delta \rho \beta \sqrt{\mathcal{R}} B_{3 \mathcal{R}}^{2} \\
C_{1}^{3}(T, \mathcal{R})=0
\end{array}\right]=0 \\
& \left\{\begin{array}{c}
C_{2 t}^{3}-\beta\left(\mathcal{R}+\omega_{4}\right) C_{2}^{3}+\frac{3}{2} \beta^{2} \mathcal{P}_{1} C_{6}^{3}-2 \mathcal{P}_{4} \delta \rho \beta \sqrt{\mathcal{R}} B_{4 \mathcal{R}}^{2}-\omega_{6} B_{1 \mathcal{R}}^{2}=0 \\
C_{2}^{3}(T, \mathcal{R})=0
\end{array}\right. \\
& \left\{\begin{array}{c}
C_{3 t}^{3}-\beta\left(\mathcal{R}+\omega_{5}\right) C_{3}^{3}+\frac{3}{2} \beta^{2} \mathcal{P}_{2} B_{7}^{2}-2 \mathcal{P}_{5} \delta \rho \beta \sqrt{\mathcal{R}} B_{5 \mathcal{R}}^{2}-\omega_{7} B_{1 \mathcal{R}}^{2}=0 \\
C_{3}^{3}(T, \mathcal{R})=0
\end{array}\right. \\
& \left\{\begin{array}{c}
C_{4 t}^{3}-2 \beta\left(\mathcal{R}+\omega_{4}\right) C_{4}^{3}+2 \mathcal{P}_{1}\left(2 \beta^{2}+\beta(2 \beta+1) C_{8}^{3}+(a-b \mathcal{R}) A_{2 \mathcal{R}}^{1}+\frac{1}{2} \delta^{2} \mathcal{R} A_{2 \mathcal{R} \mathcal{R}}^{1}\right. \\
-\frac{1}{2} \theta \delta^{2} \mathcal{R}\left(1-\mathcal{P}_{11} \rho^{2}\right) A_{1 \mathcal{R}}^{1} A_{2 \mathcal{R}}^{1}-3 \mathcal{P}_{4} \delta \rho \beta \sqrt{\mathcal{R}} B_{6 \mathcal{R}}^{2}-\omega_{6} B_{2 \mathcal{R}}^{2}+\frac{\omega_{2}}{2 \theta}=0 \\
C_{4}^{3}(T, \mathcal{R})=0
\end{array}\right. \\
& \left\{\begin{array}{c}
C_{5 t}^{3}-2 \beta\left(\mathcal{R}+\omega_{5}\right) C_{5}^{3}+2 \mathcal{P}_{2}\left(2 \beta^{2}+\beta(2 \beta+1) C_{9}^{3}+(a-b \mathcal{R}) A_{3 \mathcal{R}}^{1}+\frac{1}{2} \delta^{2} \mathcal{R} A_{3 \mathcal{R} \mathcal{R}}^{1}\right. \\
-\frac{1}{2} \theta \delta^{2} \mathcal{R}\left(1-\mathcal{P}_{11} \rho^{2}\right) A_{1 \mathcal{R}}^{1} A_{3 \mathcal{R}}^{1}-3 \mathcal{P}_{5} \delta \rho \beta \sqrt{\mathcal{R}} B_{7 \mathcal{R}}^{2}-\omega_{7} B_{3 \mathcal{R}}^{2}+\frac{\omega_{3}}{2 \theta}=0 \\
C_{5}^{3}(T, \mathcal{R})=0
\end{array}\right. \\
& \left\{\begin{array}{c}
C_{6 t}^{3}-3 \beta\left(\mathcal{R}+\omega_{4}\right) C_{6}^{3}+\omega_{6} B_{4 \mathcal{R}}^{2}=0 \\
C_{6}^{3}(T, \mathcal{R})=0
\end{array},\right.
\end{aligned}
$$




$$
\begin{gathered}
\left\{\begin{array}{c}
C_{7 t}^{3}-3 \beta\left(\mathcal{R}+\omega_{5}\right) C_{7}^{3}+\omega_{7} B_{5 \mathcal{R}}^{2}=0 \\
C_{7}^{2}(T, \mathcal{R})=0
\end{array}\right. \\
\left\{\begin{array}{c}
C_{8 t}^{3}-2 \beta\left(\mathcal{R}+\omega_{4}\right) C_{8}^{2}-\frac{1}{2} \theta \delta^{2} \mathcal{R}\left(1-\mathcal{P}_{11} \rho^{2}\right)\left(A_{2 \mathcal{R}}^{1}\right)^{2}-\omega_{6} B_{6 \mathcal{R}}^{2}=0 \\
C_{4}^{3}(T, \mathcal{R})=0
\end{array}\right. \\
\left\{\begin{array}{c}
C_{9 t}^{2}-2 \beta\left(\mathcal{R}+\omega_{5}\right) C_{9}^{2}-\frac{1}{2} \theta \delta^{2} \mathcal{R}\left(1-\mathcal{P}_{11} \rho^{2}\right)\left(A_{3 \mathcal{R}}^{1}\right)^{2}-\omega_{7} B_{7 \mathcal{R}}^{2}=0 \\
C_{5}^{2}(T, \mathcal{R})=0
\end{array}\right.
\end{gathered}
$$

Solving (4.48) - (4.56), we obtain

$$
\begin{aligned}
& C_{1}^{3}(t, \mathcal{R})=\left[\begin{array}{c}
\frac{1}{2} \theta \delta^{2} \mathcal{R}\left(1-\mathcal{P}_{11} \rho^{2}\right) \int_{t}^{T}\left(A_{1 \mathcal{R}}^{1}\right)^{2} d \tau+2 \mathcal{P}_{4} \delta \rho \beta \sqrt{\mathcal{R}} \int_{t}^{T} B_{2 \mathcal{R}}^{2} d \tau \tau \\
+2 \mathcal{P}_{5} \delta \rho \beta \sqrt{\mathcal{R}} \int_{t}^{T} B_{3 \mathcal{R}}^{2} d-\mathcal{P}_{1} \beta(2 \beta+1) \int_{t}^{T} C_{4}^{3} d \tau \\
-\mathcal{P}_{2} \beta(2 \beta+1) \int_{t}^{T} C_{5}^{3} d \tau-(a-b \mathcal{R}) \int_{t}^{T} A_{1 \mathcal{R}}^{1} d \tau-\frac{1}{2} \delta^{2} \mathcal{R} \int_{t}^{T} A_{1 \mathcal{R} R}^{1} d \tau
\end{array}\right] \\
& C_{2}^{3}(t, \mathcal{R})=e^{\beta\left(\mathcal{R}+\omega_{4}\right)(t-T)}\left[\begin{array}{c}
2 \mathcal{P}_{4} \delta \rho \beta \sqrt{\mathcal{R}} \int_{t}^{T} B_{4 \mathcal{R}}^{2} e^{\beta\left(\mathcal{R}+\omega_{4}\right)(T-\tau)} d \tau \\
+\omega_{6} \int_{t}^{T} B_{1 \mathcal{R}}^{2} e^{\beta\left(\mathcal{R}+\omega_{4}\right)(T-\tau)} d \tau \\
-\frac{3}{2} \beta^{2} \mathcal{P}_{1} \int_{t}^{T} C_{6}^{3} e^{\beta\left(\mathcal{R}+\omega_{4}\right)(T-\tau)} d \tau \\
2 \mathcal{P}_{5} \delta \rho \beta \sqrt{\mathcal{R}} \int_{t}^{T} B_{5 \mathcal{R}}^{2} e^{\beta\left(\mathcal{R}+\omega_{5}\right)(T-\tau)} d \tau \\
+\omega_{7} \int_{t}^{T} B_{1 \mathcal{R}}^{2} e^{\beta\left(\mathcal{R}+\omega_{5}\right)(T-\tau)} d \tau \\
-\frac{3}{2} \beta^{2} \mathcal{P}_{2} \int_{t}^{T} C_{7}^{2} e^{\beta\left(\mathcal{R}+\omega_{5}\right)(T-\tau)} d \tau
\end{array}\right]
\end{aligned}
$$

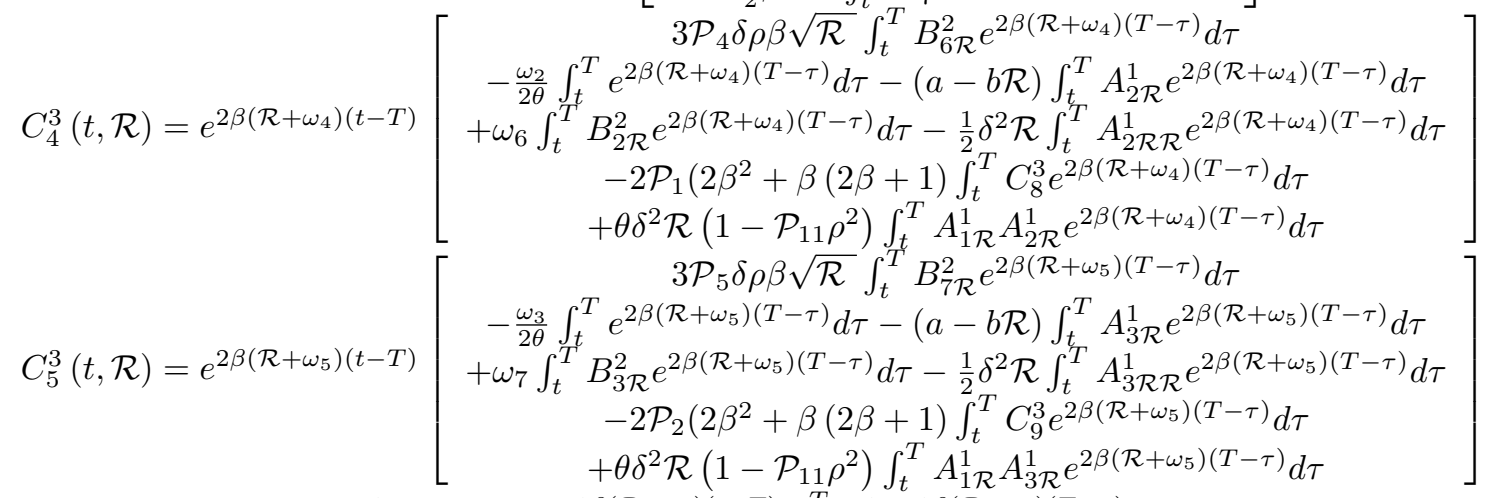

$$
\begin{aligned}
& C_{6}^{3}(t, \mathcal{R})=\omega_{6} e^{3 \beta\left(\mathcal{R}+\omega_{4}\right)(t-T)} \int_{t}^{T} B_{4 \mathcal{R}}^{1} e^{3 \beta\left(\mathcal{R}+\omega_{4}\right)(T-\tau)} d \tau \\
& C_{7}^{3}(t, \mathcal{R})=\omega_{7} e^{3 \beta\left(\mathcal{R}+\omega_{5}\right)(t-T)} \int_{t}^{T} B_{5 \mathcal{R}}^{1} e^{3 \beta\left(\mathcal{R}+\omega_{5}\right)(T-\tau)} d \tau
\end{aligned}
$$

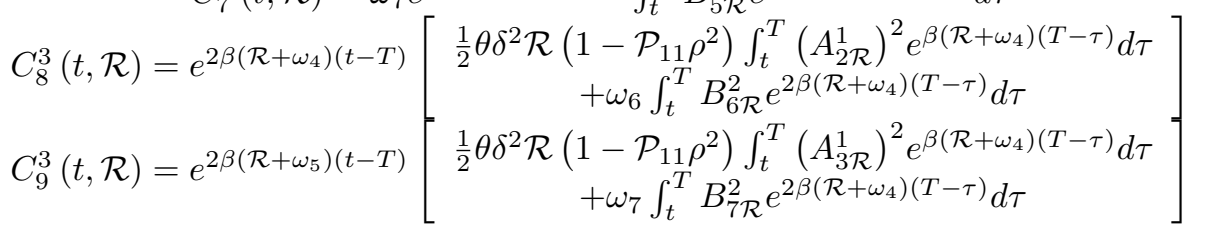

Substituting (4.57) into (4.46), we obtain 


$$
\begin{aligned}
& f^{3}(t, \mathcal{R}, y, z)=\left[\begin{array}{c}
\frac{1}{2} \theta \delta^{2} \mathcal{R}\left(1-\mathcal{P}_{11} \rho^{2}\right) \int_{t}^{T}\left(A_{1 \mathcal{R}}^{1}\right)^{2} d \tau+2 \mathcal{P}_{4} \delta \rho \beta \sqrt{\mathcal{R}} \int_{t}^{T} B_{2 \mathcal{R}}^{2} d \tau \tau \\
+2 \mathcal{P}_{5} \delta \rho \beta \sqrt{\mathcal{R}} \int_{t}^{T} B_{3 \mathcal{R}}^{2} d-\mathcal{P}_{1} \beta(2 \beta+1) \int_{t}^{T} C_{4}^{3} d \tau \\
-\mathcal{P}_{2} \beta(2 \beta+1) \int_{t}^{T} C_{5}^{3} d \tau-(a-b \mathcal{R}) \int_{t}^{T} A_{1 \mathcal{R}}^{1} d \tau-\frac{1}{2} \delta^{2} \mathcal{R} \int_{t}^{T} A_{1 \mathcal{R} \mathcal{R}}^{1} d \tau
\end{array}\right] \\
& +y^{\frac{1}{2}} e^{\beta\left(\mathcal{R}+\omega_{4}\right)(t-T)}\left[\begin{array}{r}
2 \mathcal{P}_{4} \delta \rho \beta \sqrt{\mathcal{R}} \int_{t}^{T} B_{4 \mathcal{R}}^{2} e^{\beta\left(\mathcal{R}+\omega_{4}\right)(T-\tau)} \\
+\omega_{6} \int_{t}^{T} B_{1 \mathcal{R}}^{2} e^{\beta\left(\mathcal{R}+\omega_{4}\right)(T-\tau)} d \tau
\end{array}\right. \\
& -\frac{3}{2} \beta^{2} \mathcal{P}_{1} \int_{t}^{T} C_{6}^{3} e^{\beta\left(\mathcal{R}+\omega_{4}\right)(T-\tau)} d \tau \\
& +z^{\frac{1}{2}} e^{\beta\left(\mathcal{R}+\omega_{5}\right)(t-T)}\left[\begin{array}{c}
2 \mathcal{P}_{5} \delta \rho \beta \sqrt{\mathcal{R}} \int_{t}^{T} B_{5 \mathcal{R}}^{2} e^{\beta\left(\mathcal{R}+\omega_{5}\right)(T-\tau)} d \tau \\
+\omega_{7} \int_{t}^{T} B_{1 \mathcal{R}}^{2} e^{\beta\left(\mathcal{R}+\omega_{5}\right)(T-\tau)} d \tau \\
-\frac{3}{2} \beta^{2} \mathcal{P}_{2} \int_{t}^{T} C_{7}^{2} e^{\beta\left(\mathcal{R}+\omega_{5}\right)(T-\tau)} d \tau
\end{array}\right] \\
& {\left[3 \mathcal{P}_{4} \delta \rho \beta \sqrt{\mathcal{R}} \int_{t}^{T} B_{6 \mathcal{R}}^{2} e^{2 \beta\left(\mathcal{R}+\omega_{4}\right)(T-\tau)} d \tau\right.} \\
& +y e^{2 \beta\left(\mathcal{R}+\omega_{4}\right)(t-T)} \quad \begin{array}{c}
-\frac{\omega_{2}}{2 \theta} \int_{t}^{T} e^{2 \beta\left(\mathcal{R}+\omega_{4}\right)(T-\tau)} d \tau-(a-b \mathcal{R}) \int_{t}^{T} A_{2 \mathcal{R}}^{1} e^{2 \beta\left(\mathcal{R}+\omega_{4}\right)(T-\tau)} d \tau \\
+\omega_{6} \int_{t}^{T} B_{2 \mathcal{R}}^{2} e^{2 \beta\left(\mathcal{R}+\omega_{4}\right)(T-\tau)} d \tau-\frac{1}{2} \delta^{2} \mathcal{R} \int_{t}^{T} A_{2 \mathcal{R} \mathcal{R}}^{1} e^{2 \beta\left(\mathcal{R}+\omega_{4}\right)(T-\tau)} d \tau
\end{array} \\
& -2 \mathcal{P}_{1}\left(2 \beta^{2}+\beta(2 \beta+1) \int_{t}^{T} C_{8}^{3} e^{2 \beta\left(\mathcal{R}+\omega_{4}\right)(T-\tau)} d \tau\right. \\
& +\theta \delta^{2} \mathcal{R}\left(1-\mathcal{P}_{11} \rho^{2}\right) \int_{t}^{T} A_{1 \mathcal{R}}^{1} A_{2 \mathcal{R}}^{1} e^{2 \beta\left(\mathcal{R}+\omega_{4}\right)(T-\tau)} d \tau \\
& +z e^{2 \beta\left(\mathcal{R}+\omega_{5}\right)(t-T)}\left[\begin{array}{c}
3 \mathcal{P}_{5} \delta \rho \beta \sqrt{\mathcal{R}} \int_{t}^{T} B_{7 \mathcal{R}}^{2} e^{2 \beta\left(\mathcal{R}+\omega_{5}\right)(T-\tau)} d \tau \\
-\frac{\omega_{3}}{2 \theta} \int_{t}^{T} e^{2 \beta\left(\mathcal{R}+\omega_{5}\right)(T-\tau)} d \tau-(a-b \mathcal{R}) \int_{t}^{T} A_{3 \mathcal{R}}^{1} e^{2 \beta\left(\mathcal{R}+\omega_{5}\right)(T-\tau)} d \tau \\
+\omega_{7} \int_{t}^{T} B_{3 \mathcal{R}}^{2} e^{2 \beta\left(\mathcal{R}+\omega_{5}\right)(T-\tau)} d \tau-\frac{1}{2} \delta^{2} \mathcal{R} \int_{t}^{T} A_{3 \mathcal{R} \mathcal{R}}^{1} e^{2 \beta\left(\mathcal{R}+\omega_{5}\right)(T-\tau)} d \tau
\end{array}\right. \\
& -2 \mathcal{P}_{2}\left(2 \beta^{2}+\beta(2 \beta+1) \int_{t}^{T} C_{9}^{3} e^{2 \beta\left(\mathcal{R}+\omega_{5}\right)(T-\tau)} d \tau\right. \\
& +\theta \delta^{2} \mathcal{R}\left(1-\mathcal{P}_{11} \rho^{2}\right) \int_{t}^{T} A_{1 \mathcal{R}}^{1} A_{3 \mathcal{R}}^{1} e^{2 \beta\left(\mathcal{R}+\omega_{5}\right)(T-\tau)} d \tau \\
& +y^{\frac{3}{2}} \omega_{6} e^{3 \beta\left(\mathcal{R}+\omega_{4}\right)(t-T)} \int_{t}^{T} B_{4 \mathcal{R}}^{1} e^{3 \beta\left(\mathcal{R}+\omega_{4}\right)(T-\tau)} d \tau \\
& +z^{\frac{3}{2}} \omega_{7} e^{3 \beta\left(\mathcal{R}+\omega_{5}\right)(t-T)} \int_{t}^{T} B_{5 \mathcal{R}}^{1} e^{3 \beta\left(\mathcal{R}+\omega_{5}\right)(T-\tau)} d \tau
\end{aligned}
$$

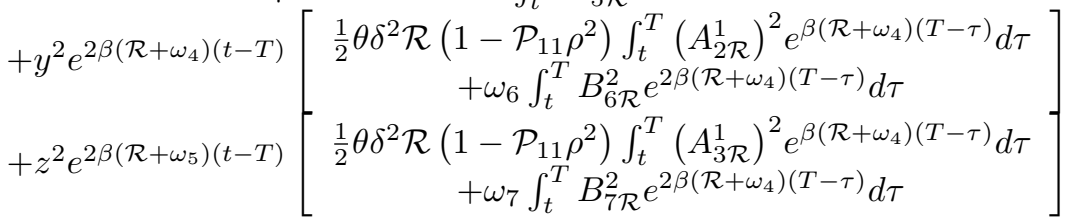

Therefore, from (4.18), we have

$$
f_{\varepsilon}(t, \mathcal{R}, y, z)=f^{1}(t, \mathcal{R}, y, z)+\sqrt{\varepsilon} f^{2}(t, \mathcal{R}, y, z)+\varepsilon f^{3}(t, \mathcal{R}, y, z)
$$

where $f^{1}(t, \mathcal{R}, y, z), f^{2}(t, \mathcal{R}, y, z)$ and $f^{3}(t, \mathcal{R}, y, z)$ are given in equation (4.34), (4.45) and (4.58) respectively.

Hence, lemma 4.1 is proved.

Lemma 4.2. The optimal value function is given as

$$
\mathcal{N}\left(t, \mathcal{R}, s_{1}, s_{2}, h\right)=-\frac{1}{\theta} e^{-\theta\left(f_{\varepsilon}(t, \mathcal{R}, y, z)+h g(t)\right)}
$$

where

$$
\begin{gathered}
f_{\varepsilon}(t, \mathcal{R}, y, z)=f^{1}(t, \mathcal{R}, y, z)+\sqrt{\varepsilon} f^{2}(t, \mathcal{R}, y, z)+\varepsilon f^{3}(t, \mathcal{R}, y, z) \\
g(t)=\operatorname{Exp}\left[\mathcal{R}+\frac{\mathcal{P}_{6}\left(\left(\mathcal{P}_{1} \mathcal{P}_{2}-\mathcal{P}_{3}^{2}\right) \alpha\right.}{2 \mathcal{P}_{3}\left(2 m_{1} m_{2}-m_{1} \mathcal{R}-m_{2} \mathcal{R}\right)}\right](T-t)
\end{gathered}
$$

Proof. Substituting equation (4.8) and lemma 4.1 into (4.2), then lemma 4.2 is proved. 
Lemma 4.3. The optimal investment plans are given as

$$
\begin{aligned}
& \varphi_{1}^{*}=\frac{1}{h}\left[\begin{array}{c}
\frac{\left[\mathcal{P}_{3} s_{1}^{\beta}\left(m_{2}-\mathcal{R}\right)-\mathcal{P}_{2} s_{2}^{\beta}\left(m_{1}-\mathcal{R}\right)\right]}{\theta\left(\mathcal{P}_{1} \mathcal{P}_{2}-\mathcal{P}_{3}^{2}\right) s_{1}^{2 \beta} s_{2}^{\beta}} \\
+\frac{2 \beta}{s_{1}^{2 \beta}} f_{y}+\frac{\left(\mathcal{P}_{3} \mathcal{P}_{5}-\mathcal{P}_{2} \mathcal{P}_{4}\right) \sqrt{\mathcal{R}} \delta \rho}{\left(\mathcal{P}_{1} \mathcal{P}_{2}-\mathcal{P}_{3}^{2}\right) s_{1}^{\beta}} f_{\mathcal{R}}
\end{array}\right] \operatorname{Exp}\left[\mathcal{R}+\frac{\mathcal{P}_{6}\left(\left(\mathcal{P}_{1} \mathcal{P}_{2}-\mathcal{P}_{3}^{2}\right) \alpha\right.}{2 \mathcal{P}_{3}\left(2 m_{1} m_{2}-m_{1} \mathcal{R}-m_{2} \mathcal{R}\right)}\right](t-T) \\
& \varphi_{2}^{*}=\frac{1}{h}\left[\begin{array}{c}
\frac{\left[\mathcal{P}_{3} s_{2}^{\beta}\left(m_{1}-\mathcal{R}\right)-\mathcal{P}_{1} s_{1}^{\beta}\left(m_{2}-\mathcal{R}\right)\right]}{\theta\left(\mathcal{P}_{1} \mathcal{P}_{2}-\mathcal{P}_{3}^{2}\right) s_{1}^{2} s_{2}^{\beta}} \\
+\frac{2 \beta}{s_{2}^{2 \beta} f_{z}+\frac{\left(\mathcal{P}_{3} \mathcal{P}_{4}-\mathcal{P}_{1} \mathcal{P}_{5}\right) \sqrt{\mathcal{R}} \delta \rho}{\left(\mathcal{P}_{1} \mathcal{P}_{2}-\mathcal{P}_{3}^{2}\right) s_{2}^{\beta}} f_{\mathcal{R}}^{\beta}}
\end{array}\right] \operatorname{Exp}\left[\mathcal{R}+\frac{\mathcal{P}_{6}\left(\left(\mathcal{P}_{1} \mathcal{P}_{2}-\mathcal{P}_{3}^{2}\right) \alpha\right.}{2 \mathcal{P}_{3}\left(2 m_{1} m_{2}-m_{1} \mathcal{R}-m_{2} \mathcal{R}\right)}\right](t-T)
\end{aligned}
$$

where

$$
\begin{aligned}
& \left\{\begin{array}{c}
f_{y}=A_{2}^{1}+\sqrt{\varepsilon}\left(\frac{1}{2} y^{-\frac{1}{2}} B_{2}^{2}+B_{4}^{2}+\frac{3}{2} y^{\frac{1}{2}} B_{6}^{2}\right)+\varepsilon\left(\frac{1}{2} y^{-\frac{1}{2}} C_{2}^{3}+C_{4}^{3}+\frac{3}{2} y^{\frac{1}{2}} C_{6}^{3}+2 y C_{8}^{3}\right) \\
f_{z}=A_{3}^{1}+\sqrt{\varepsilon}\left(\frac{1}{2} z^{-\frac{1}{2}} B_{2}^{2}+B_{4}^{2}+\frac{3}{2} z^{\frac{1}{2}} B_{6}^{2}\right)+\varepsilon\left(\frac{1}{2} z^{-\frac{1}{2}} C_{2}^{3}+C_{4}^{3}+\frac{3}{2} z^{\frac{1}{2}} C_{6}^{3}+2 z C_{8}^{3}\right) \\
A_{1 \mathcal{R}}^{1}+y A_{2 \mathcal{R}}^{1}+z A_{3 \mathcal{R}}^{1} \\
f_{\mathcal{R}}=\left[\begin{array}{c}
+\sqrt{\varepsilon}\left(B_{1 \mathcal{R}}^{2}+y^{\frac{1}{2}} B_{2 \mathcal{R}}^{2}+z^{\frac{1}{2}} B_{3 \mathcal{R}}^{2}+y B_{4 \mathcal{R}}^{2}+z B_{5 \mathcal{R}}^{2}+y^{\frac{3}{2}} B_{6 \mathcal{R}}^{2}+z^{\frac{3}{2}} B_{7 \mathcal{R}}^{2}\right) \\
+\varepsilon\left(C_{1 \mathcal{R}}^{3}+y^{\frac{1}{2}} C_{2 \mathcal{R}}^{3}+z^{\frac{1}{2}} C_{3 \mathcal{R}}^{3}+y C_{4 \mathcal{R}}^{3}+z C_{5 \mathcal{R}}^{2}+y^{\frac{3}{2}} C_{6 \mathcal{R}}^{3}+z^{\frac{3}{2}} C_{7 \mathcal{R}}^{3}+y^{2} C_{8 \mathcal{R}}^{3}+z^{2} C_{9 \mathcal{R}}^{3},\right)
\end{array}\right]
\end{array}\right. \\
& \left\{\begin{array}{c}
\mathcal{P}_{1}=n_{11}^{2}+n_{12}^{2}, \quad \mathcal{P}_{2}=n_{21}^{2}+n_{22}^{2}, \mathcal{P}_{3}=n_{11} n_{21}+n_{12} n_{22} \\
\mathcal{P}_{4}=n_{11}+n_{12}, \mathcal{P}_{5}=n_{21}+n_{22}, \mathcal{P}_{6}=\frac{2 \mathcal{P}_{3}\left(m_{1}-\mathcal{R}\right)\left(m_{2}-\mathcal{R}\right)}{\left(\mathcal{P}_{1} \mathcal{P}_{2}-\mathcal{P}_{3}^{2}\right)} \\
\mathcal{P}_{7}=\frac{\mathcal{P}_{2}\left(m_{1}-\mathcal{R}\right)^{2}}{\left(\mathcal{P}_{1} \mathcal{P}_{2}-\mathcal{P}_{3}^{2}\right)}, \mathcal{P}_{8}=\frac{\mathcal{P}_{1}\left(m_{2}-\mathcal{R}\right)^{2}}{\left(\mathcal{P}_{1} \mathcal{P}_{2}-\mathcal{P}_{3}^{2}\right)}, \mathcal{P}_{9}=\frac{\left(\mathcal{P}_{2} \mathcal{P}_{4}-\mathcal{P}_{3} \mathcal{P}_{5}\right)}{\left(\mathcal{P}_{1} \mathcal{P}_{2}-\mathcal{P}_{3}^{2}\right)} \\
\mathcal{P}_{10}=\frac{\left(\mathcal{P}_{1} \mathcal{P}_{5}-\mathcal{P}_{3} \mathcal{P}_{4}\right)}{\left(\mathcal{P}_{1} \mathcal{P}_{2}-\mathcal{P}_{3}^{2}\right)}, \mathcal{P}_{11}=\frac{\left(\mathcal{P}_{2} \mathcal{P}_{4}^{2}+\mathcal{P}_{1} \mathcal{P}_{5}^{2}-2 \mathcal{P}_{3} \mathcal{P}_{4} \mathcal{P}_{5}\right)}{\left(\mathcal{P}_{1} \mathcal{P}_{2}-\mathcal{P}_{3}^{2}\right)}
\end{array}\right.
\end{aligned}
$$

Proof. Recall from equation (3.7) and (3.8), we have

$$
\begin{aligned}
& \varphi_{1}^{*}=\frac{\left[\mathcal{P}_{3} s_{1}^{\beta}\left(m_{2}-\mathcal{R}\right)-\mathcal{P}_{2} s_{2}^{\beta}\left(m_{1}-\mathcal{R}\right)\right]}{h\left(\mathcal{P}_{1} \mathcal{P}_{2}-\mathcal{P}_{3}^{2}\right) s_{1}^{2 \beta} s_{2}^{\beta}} \frac{\mathcal{N}_{h}}{\mathcal{N}_{h h}}-s_{1} \frac{\mathcal{N}_{h s_{1}}}{h \mathcal{N}_{h h}}-\frac{\left(\mathcal{P}_{2} \mathcal{P}_{4}-\mathcal{P}_{3} \mathcal{P}_{5}\right) \sqrt{\mathcal{R}} \delta \rho}{h\left(\mathcal{P}_{1} \mathcal{P}_{2}-\mathcal{P}_{3}^{2}\right) s_{1}^{\beta}} \frac{\mathcal{N}_{h \mathcal{R}}}{\mathcal{N}_{h h}} \\
& \varphi_{2}^{*}=\frac{\left[\mathcal{P}_{3} s_{2}^{\beta}\left(m_{1}-\mathcal{R}\right)-\mathcal{P}_{1} s_{1}^{\beta}\left(m_{2}-\mathcal{R}\right)\right]}{h\left(\mathcal{P}_{1} \mathcal{P}_{2}-\mathcal{P}_{3}^{2}\right) s_{1}^{\beta} s_{2}^{2 \beta}} \frac{\mathcal{N}_{h}}{\mathcal{N}_{h h}}-s_{2} \frac{\mathcal{N}_{h s_{2}}}{h \mathcal{N}_{h h}}-\frac{\left(\mathcal{P}_{1} \mathcal{P}_{5}-\mathcal{P}_{3} \mathcal{P}_{4}\right) \sqrt{\mathcal{R}} \delta \rho}{h\left(\mathcal{P}_{1} \mathcal{P}_{2}-\mathcal{P}_{3}^{2}\right) s_{2}^{\beta}} \frac{\mathcal{N}_{h \mathcal{R}}}{\mathcal{N}_{h h}}
\end{aligned}
$$

From equation (4.3), (4.8) and (4.10), we have

$$
\left\{\begin{array}{c}
\frac{\mathcal{N}_{h}}{\mathcal{N}_{h h}}=-\frac{1}{\theta g}=-\frac{1}{\theta} \operatorname{Exp}\left[\mathcal{R}+\frac{\mathcal{P}_{6}\left(\left(\mathcal{P}_{1} \mathcal{P}_{2}-\mathcal{P}_{3}^{2}\right) \alpha\right.}{2 \mathcal{P}_{3}\left(2 m_{1} m_{2}-m_{1} \mathcal{R}-m_{2} \mathcal{R}\right)}\right](t-T) \\
\frac{\mathcal{N}_{h s_{1}}}{\mathcal{N}_{h h}}=\frac{v_{s_{1}}}{g}=-2 \beta s_{1}^{-2 \beta-1} f_{y} \operatorname{Exp}\left[\mathcal{R}+\frac{\mathcal{P}_{6}\left(\left(\mathcal{P}_{1} \mathcal{P}_{2}-\mathcal{P}_{3}^{2}\right) \alpha\right.}{2 \mathcal{P}_{3}\left(2 m_{1} m_{2}-m_{1} \mathcal{R}-m_{2} \mathcal{R}\right)}\right](t-T) \\
\frac{\mathcal{N}_{h s_{2}}}{\mathcal{N}_{h h}}=\frac{w_{s_{2}}}{g}=-2 \beta s_{2}^{-2 \beta-1} f_{z} \operatorname{Exp}\left[\mathcal{R}+\frac{\mathcal{P}_{6}\left(\left(\mathcal{P}_{1} \mathcal{P}_{2}-\mathcal{P}_{3}^{2}\right) \alpha\right.}{2 \mathcal{P}_{3}\left(2 m_{1} m_{2}-m_{1} \mathcal{R}-m_{2} \mathcal{R}\right)}\right](t-T) \\
\frac{\mathcal{N}_{h \mathcal{R}}}{\mathcal{N}_{h h}}=\frac{\left(v_{\mathcal{R}}+w_{\mathcal{R}}\right)}{g}=f_{\mathcal{R}} \operatorname{Exp}\left[\mathcal{R}+\frac{\mathcal{P}_{6}\left(\left(\mathcal{P}_{1} \mathcal{P}_{2}-\mathcal{P}_{3}^{2}\right) \alpha\right.}{2 \mathcal{P}_{3}\left(2 m_{1} m_{2}-m_{1} \mathcal{R}-m_{2} \mathcal{R}\right)}\right](t-T)
\end{array}\right.
$$

where

$$
\left\{\begin{array}{c}
f_{y}=A_{2}^{1}+\sqrt{\varepsilon}\left(\frac{1}{2} y^{-\frac{1}{2}} B_{2}^{2}+B_{4}^{2}+\frac{3}{2} y^{\frac{1}{2}} B_{6}^{2}\right)+\varepsilon\left(\frac{1}{2} y^{-\frac{1}{2}} C_{2}^{3}+C_{4}^{3}+\frac{3}{2} y^{\frac{1}{2}} C_{6}^{3}+2 y C_{8}^{3}\right) \\
f_{z}=A_{3}^{1}+\sqrt{\varepsilon}\left(\frac{1}{2} z^{-\frac{1}{2}} B_{2}^{2}+B_{4}^{2}+\frac{3}{2} z^{\frac{1}{2}} B_{6}^{2}\right)+\varepsilon\left(\frac{1}{2} z^{-\frac{1}{2}} C_{2}^{3}+C_{4}^{3}+\frac{3}{2} z^{\frac{1}{2}} C_{6}^{3}+2 z C_{8}^{3}\right) \\
A_{1 \mathcal{R}}^{1}+y A_{2 \mathcal{R}}^{1}+z A_{3 \mathcal{R}}^{1} \\
f_{\mathcal{R}}=\left[\begin{array}{c}
+\sqrt{\varepsilon}\left(B_{1 \mathcal{R}}^{2}+y^{\frac{1}{2}} B_{2 \mathcal{R}}^{2}+z^{\frac{1}{2}} B_{3 \mathcal{R}}^{2}+y B_{4 \mathcal{R}}^{2}+z B_{5 \mathcal{R}}^{2}+y^{\frac{3}{2}} B_{6 \mathcal{R}}^{2}+z^{\frac{3}{2}} B_{7 \mathcal{R}}^{2}\right) \\
+\varepsilon\left(C_{1 \mathcal{R}}^{3}+y^{\frac{1}{2}} C_{2 \mathcal{R}}^{3}+z^{\frac{1}{2}} C_{3 \mathcal{R}}^{3}+y C_{4 \mathcal{R}}^{3}+z C_{5 \mathcal{R}}^{2}+y^{\frac{3}{2}} C_{6 \mathcal{R}}^{3}+z^{\frac{3}{2}} C_{7 \mathcal{R}}^{3}+y^{2} C_{8 \mathcal{R}}^{3}+z^{2} C_{9 \mathcal{R}}^{3},\right)
\end{array}\right]
\end{array}\right.
$$

Substituting (4.62) into (3.7) and (3.8), this completes the proof. 
Remark 4.4. If the risk free interest rate $\mathcal{R}$ is not stochastic, i.e. $\varepsilon=0$ and $\rho=0$, then

$$
\begin{aligned}
& \varphi_{1}^{*}=\frac{1}{h}\left[\begin{array}{c}
\frac{\left[\mathcal{P}_{3} s_{1}^{\beta}\left(m_{2}-\mathcal{R}\right)-\mathcal{P}_{2} s_{2}^{\beta}\left(m_{1}-\mathcal{R}\right)\right]}{\theta\left(\mathcal{P}_{1} \mathcal{P}_{2}-\mathcal{P}_{3}^{2}\right) s_{1}^{2 \beta} s_{2}^{\beta}}+\frac{2 \beta}{s_{1}^{2 \beta}} A_{2}^{1} \\
-\frac{\left(\mathcal{P}_{2} \mathcal{P}_{4}-\mathcal{P}_{3} \mathcal{P}_{5}\right) \sqrt{\mathcal{R}} \delta \rho}{\left(\mathcal{P}_{1} \mathcal{P}_{2}-\mathcal{P}_{3}^{2}\right) s_{1}^{\beta}}\left(A_{1 \mathcal{R}}^{1}+y A_{2 \mathcal{R}}^{1}+z A_{3 \mathcal{R}}^{1}\right)
\end{array}\right] \operatorname{Exp}\left[\mathcal{R}+\frac{\mathcal{P}_{6}\left(\left(\mathcal{P}_{1} \mathcal{P}_{2}-\mathcal{P}_{3}^{2}\right) \alpha\right.}{2 \mathcal{P}_{3}\left(2 m_{1} m_{2}-m_{1} \mathcal{R}-m_{2} \mathcal{R}\right)}\right](t-T) \\
& \varphi_{2}^{*}=\frac{1}{h}\left[\begin{array}{c}
\frac{\left[\mathcal{P}_{3} s_{2}^{\beta}\left(m_{1}-\mathcal{R}\right)-\mathcal{P}_{1} s_{1}^{\beta}\left(m_{2}-\mathcal{R}\right)\right]}{\theta\left(\mathcal{P}_{1} \mathcal{P}_{2}-\mathcal{P}_{3}^{2}\right) s_{1}^{\beta \beta} s_{2}^{\beta}}+\frac{2 \beta}{s_{2}^{2 \beta}} A_{3}^{1} \\
-\frac{\left(\mathcal{P}_{1} \mathcal{P}_{5}-\mathcal{P}_{3} \mathcal{P}_{4}\right) \sqrt{\mathcal{R}} \delta \rho}{\left(\mathcal{P}_{1} \mathcal{P}_{2}-\mathcal{P}_{3}^{2}\right) s_{2}^{\beta}}\left(A_{1 \mathcal{R}}^{1}+y A_{2 \mathcal{R}}^{1}+z A_{3 \mathcal{R}}^{1}\right)
\end{array}\right] \operatorname{Exp}\left[\mathcal{R}+\frac{\mathcal{P}_{6}\left(\left(\mathcal{P}_{1} \mathcal{P}_{2}-\mathcal{P}_{3}^{2}\right) \alpha\right.}{2 \mathcal{P}_{3}\left(2 m_{1} m_{2}-m_{1} \mathcal{R}-m_{2} \mathcal{R}\right)}\right](t-T)
\end{aligned}
$$

where

$$
A_{1}^{1}(t, \mathcal{R})=\left[\begin{array}{c}
{\left[\frac{\mathcal{P}_{1}(2 \beta+1) \omega_{2}}{4 \theta\left(\mathcal{R}+\omega_{4}\right)}+\frac{\mathcal{P}_{2}(2 \beta+1) \omega_{3}}{4 \theta\left(\mathcal{R}+\omega_{5}\right)}\right](T-t)-\frac{\mathcal{P}_{1}(2 \beta+1) \omega_{2}}{4 \beta \theta\left(\mathcal{R}+\omega_{4}\right)^{2}}\left[1-e^{2 \beta\left(\mathcal{R}+\omega_{4}\right)(t-T)}\right]} \\
-\frac{\mathcal{P}_{1}(2 \beta+1) \omega_{2}}{4 \beta \theta\left(\mathcal{R}+\omega_{4}\right)^{2}}\left[1-e^{2 \beta\left(\mathcal{R}+\omega_{4}\right)(t-T)}\right]
\end{array}\right]
$$

\section{$5 \quad$ Sensitivity Analysis}

Here we present some numerical simulations to study the effects of some parameters on the optimal investment plan under logarithm utility. To achieve this, the following data will be used unless otherwise stated $n_{11}=1, \quad n_{12}=0.9, n_{21}=0.85, \quad n_{11}=0.8, \beta=-1, m_{1}=0.4, m_{2}=0.3, h=$ $1, \alpha=1, \rho=-0.5, \mathcal{R}(0)=0.05, \mathcal{S}_{1}(0)=1.5, \mathcal{S}_{2}(0)=1.2, T=3$

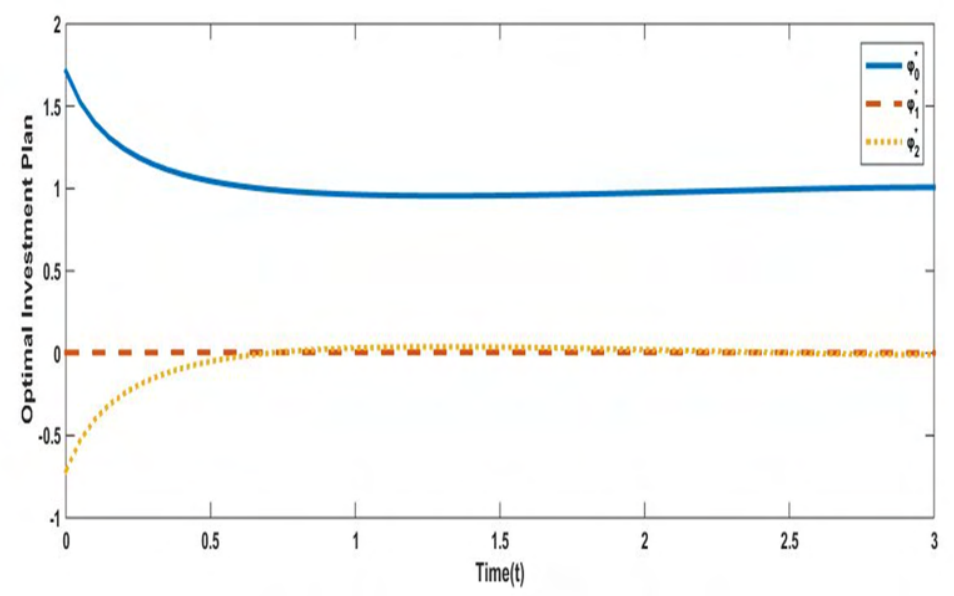

Evolution of the optimal investment plan $\varphi_{0}^{*}, \varphi_{1}^{*}$, and $\varphi_{2}^{*}$ 


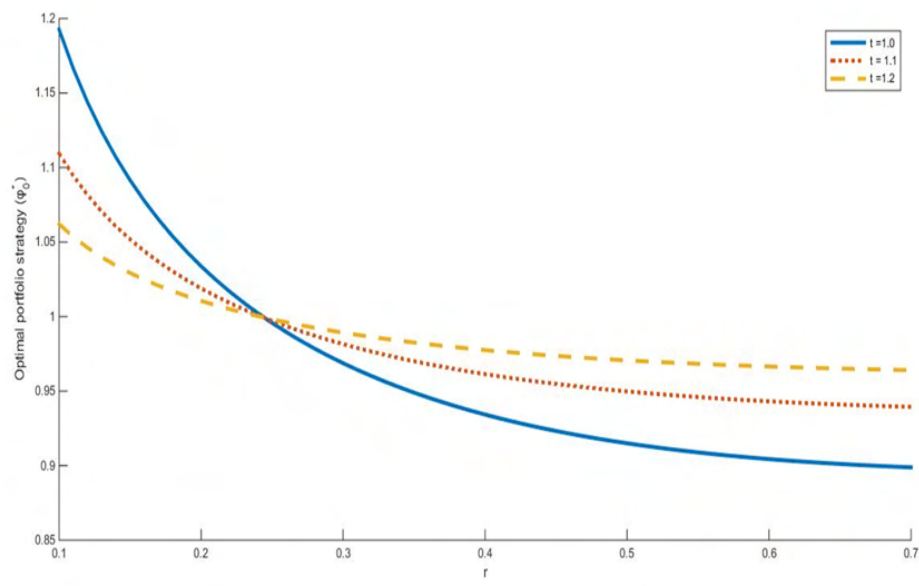

The impact of the risk free interest $r$ on $\varphi_{1}^{*}$

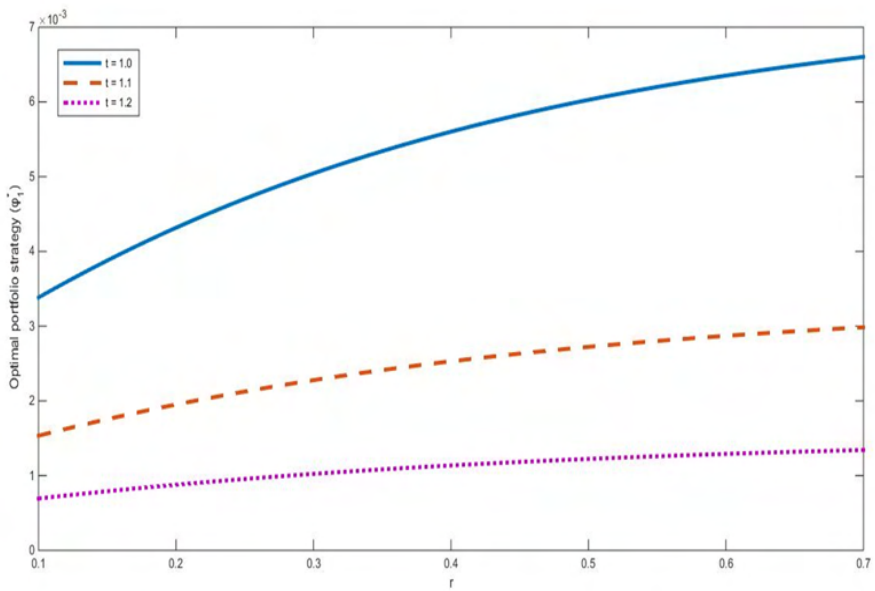

The impact of the risk free interest $r$ on $\varphi_{0}^{*}$

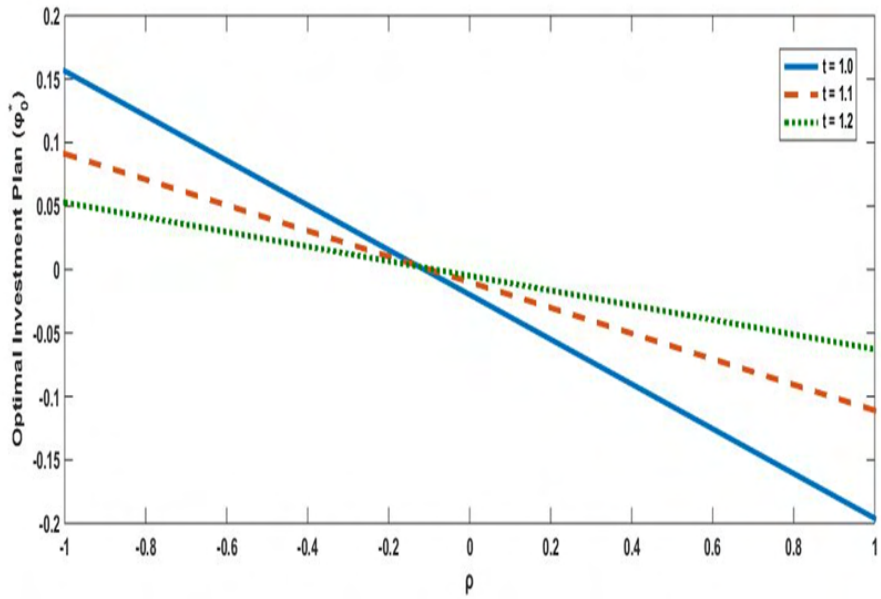

The impact of the correlation coefficient $\rho$ on $\varphi_{0}^{*}$ 


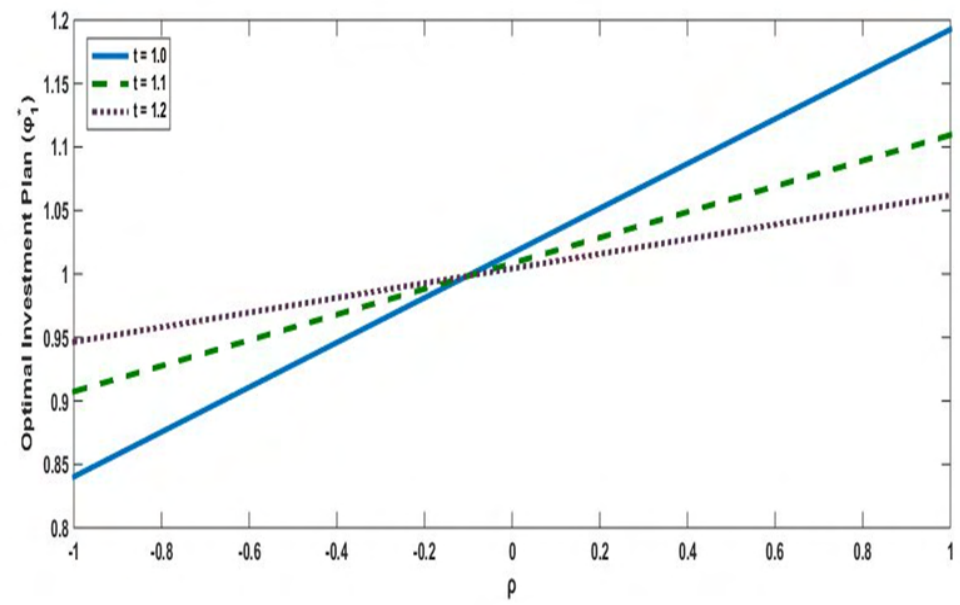

The impact of the correlation coefficient $\rho$ on $\varphi_{1}^{*}$

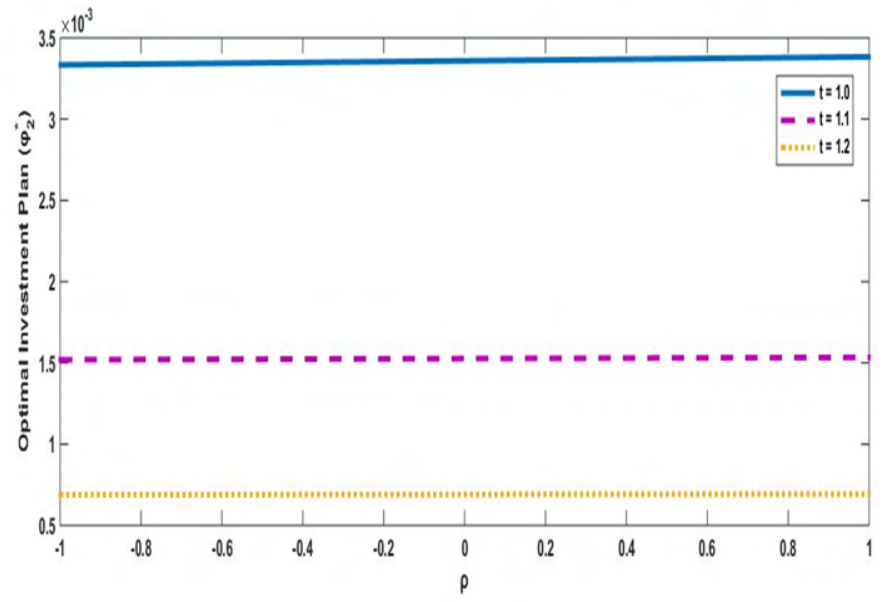

The impact of the correlation coefficient $\rho$ on $\varphi_{2}^{*}$

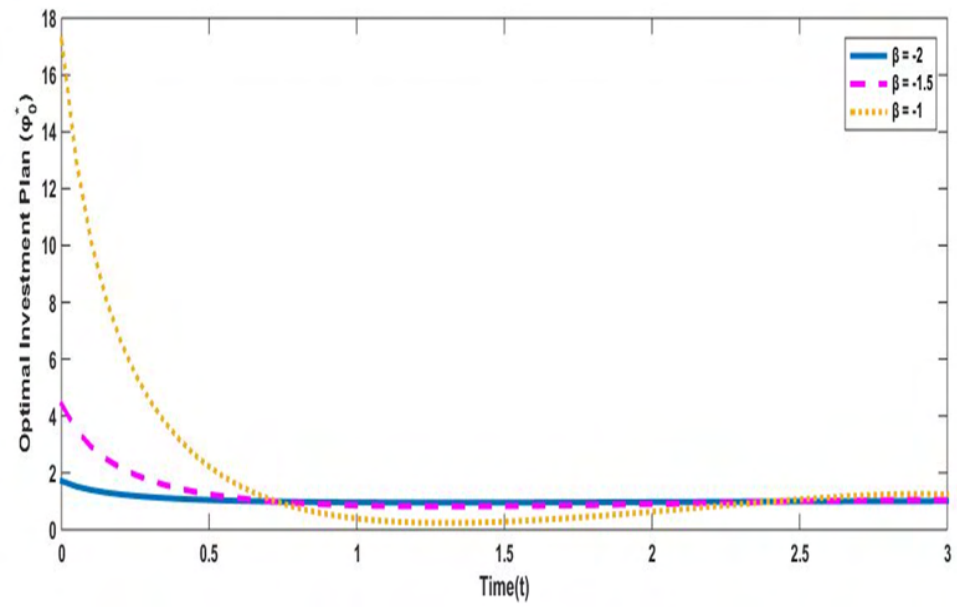

Evolution of $\varphi_{0}^{*}$ with different elasticity parameter $\beta$ 


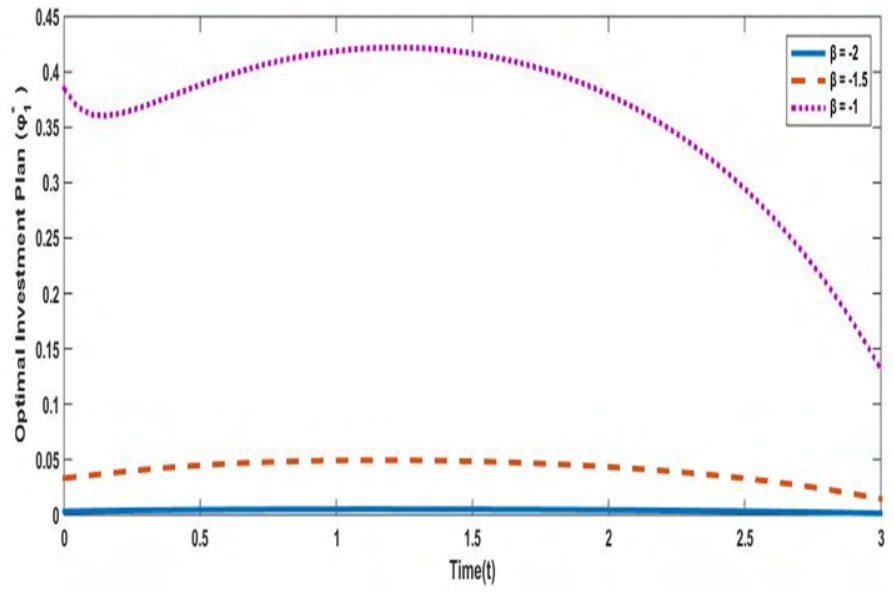

Evolution of $\varphi_{1}^{*}$ with different elasticity parameter $\beta$

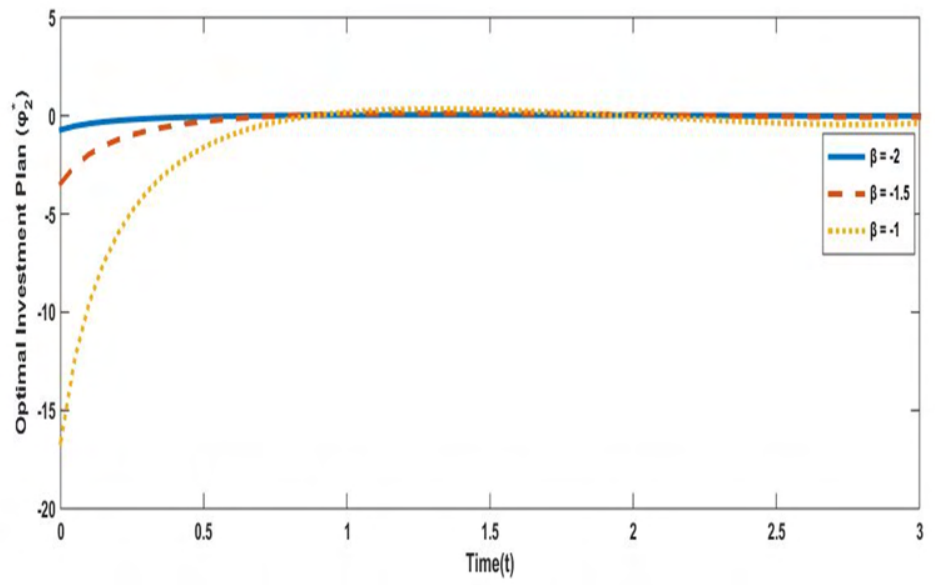

Evolution of $\varphi_{2}^{*}$ with different elasticity parameter $\beta$

\section{Discussion}

The impact of sensitive parameters on the optimal investment plan is analysed. In Figure 5, the simulation of optimal investment plan of the three assets is given against time; the graph shows that at the initial time, the investor will invest more in the risk free asset and less in the other two risky assets and as expiration date draws closer the investor will begin to invest more in risky and reduce investment in risk free asset. In figure 5 and 5 , the investment plan for the risk free asset increases with interest rate increases while that of the risky asset decreases with interest rate. This is because the investors prefer investment in risk free asset than risky asset whenever the interest rate appreciates since it is risk-less. Figure 5,5 , and 5 give the analysis of the impact of the correlation coefficient $\rho$ on the optimal investment plans $\varphi_{0}^{*}, \varphi_{1}^{*}$ and $\varphi_{2}^{*}$, it is observed that as $\rho$ increases $\varphi_{0}^{*}$ decreases, $\varphi_{1}^{*}$ increases significantly while $\varphi_{2}^{*}$ experience little or no increase. Also, we observed also that as the investment time draws closer to it expiring date, the insurer will invest less in the risky assets and more in the risk free asset. Figure 5 , shows that as $\beta$ reduces, $\varphi_{0}^{*}$ increases which implies that investors with high $\theta$ may invest more in risk free asset to prevent more loss especially when the market is very volatile. On the contrary, figures 5 and 5 , show that as $\beta$ reduces, the optimal investment plan for the two risky assets decreases which implies that investors with high $\theta$ may be more scared to invest in the risky assets when the market is highly volatile. Furthermore, 
at the initial stage of investment we observed a huge disparity between the investment plan when $\beta=-1$ when compared to $\beta=-1.5$ and -2 . This confirm that the choice of $\beta=-1$ could be most suitable in choosing an investment plan.

From equation (4.60) and (4.61), the optimal investment plan decreases with an increase in the initial wealth. Also, from remark 4.4, the optimal investment plan for the two risky assets reduce to the result in [27] when $\mathcal{R}$ is not stochastic.

\section{Conclusion}

This paper investigated explicit solutions of the optimal investment plans of an investor with exponential utility function exhibiting CARA under CEV model and stochastic interest rate. We considered a portfolio with risk-free asset modelled by Cox- Ingersoll-Ross (CIR) process and two risky assets modelled by the CEV process. The power transformation and change of variable approach with asymptotic expansion technique was used to determine explicit solutions of the optimal investment plans. Furthermore, we present some numerical simulations to study the effect of the interest rate, elasticity parameter, correlation coefficient and the risk averse coefficient on the optimal investment plans.Finally, when the interest rate is constant, our result is similar to the result in [27].

\section{Acknowledgements}

The authors are grateful to the authors referred in this work.

\section{References}

[1] Merton, R. C. (1971). Optimum consumption and portfolio rules in a continuous-time model. Journal of Economic Theory, 3, 373-413.

[2] Yang, H. \& Zhang, L. (2005). Optimal investment for insurer with jump-diffusion risk process. Mathematics and Economics, 37, 615-634.

[3] Zhang, A. (2007). A secret to create a complete market from an incomplete market.Applied Mathematics and Computation, 191, 253-262.

[4] Akpanibah, E. E. \& Samaila, S. K. (2017) Stochastic strategies for optimal investment in a defined contribution (DC) pension fund, International Journal of Applied Science and Mathematical Theory, 3(??)(2017), 48-55.

[5] Karatzas, I., Lehoczky, J.P.\& Shreve, S.E., (1987). Optimal portfolio and consumption decisions for a "small investor" on a finite horizon. SIAM Journal on Control and Optimization $25,1557-1586$

[6] Pliska, S. (1986). A stochastic calculus model of continuous trading. Mathematics of Operations Research, 11, 371-382.

[7] Wang, Z., Xia, J.\& Zhang, L. (2007). Optimal investment for an insurer: the martingale approach. Mathematics and Economics, 40, 322-334.

[8] Karatzas, I. \& Shreve, S. E. (1991). Brownian Motion and Stochastic Calculus. Springer, Berlin.

[9] Boulier, J. F., Huang,S., Taillard G. (2001). Optimal management under stochastic interest rates: the case of a protected defined contribution pension fund, Insurance 28(2), 173-189.

[10] Deelstra, G., Graselli, M. \& Koehl, P. F. (2003). Optimal investment strategies in the presence of a minimum guarantee. Insurance, 33(1), 189-207. 
[11] Gao, J. (2008) Stochastic optimal control of DC pension funds, Insurance, 42(3), 1159-1164.

[12] Battocchio, P. \& Menoncin, F. (2004). Optimal pension management in a stochastic framework, Insurance 34(1), 79-95.

[13] Cairns, A. J. G., Blake, D. \& Dowd, K. (2006). Stochastic life styling: optimal dynamic asset allocation for defined contribution pension plans, Journal of Economic Dynamics and Control, $30(5), 843-877$.

[14] Akpanibah E. E., Osu, B. O., Njoku, K.N. C. \& Akak, E. O. (2017) Optimization of Wealth Investment Strategies for a DC Pension Fund with Stochastic Salary and Extra Contributions, International Journal of Partial Diff. Equations and Application, 5(1), 33-41.

[15] Njoku, K.N. C., Osu, B. O., Akpanibah, E. E.\& Ujumadu, R. N. (2017). Effect of Extra Contribution on Stochastic Optimal Investment Strategies for DC Pension with Stochastic Salary under the Affine Interest Rate Model. Journal of Mathematical Finance, 7, 821-833.

[16] Zhang C. \& Rong, X. (2013). Optimal investment strategies for DC pension with a stochastic salary under affine interest rate model. Hindawi Publishing Corporation, 2013 http://dx.doi.org/10.1155/2013/297875.

[17] Hobson, D.G. \& Rogers, L. C. G., (1998). Complete models with stochastic volatility. Mathematical Finance, 8, 27-48.

[18] Cox, J. C. \& Ross, S. A. (1976). The valuation of options for alternative stochastic processes, Journal of financial economics, 3(2, 145-166

[19] Li, D., Rong, X. \&Zhao, H. (2013). Optimal investment problem with taxes, dividends and transaction costs under the constant elasticity of variance model. Transaction on Mathematics, $12(3), 243-255$.

[20] Gu, M., Yang, Y, Li, S. \& Zhang, J. (2010). Constant elasticity of variance model for proportional reinsurance and investment strategies, Insurance: Mathematics and Economics, 46(3), $580-587$

[21] Li, D., Rong, X, . Zhao, H.\& Yi B. (2017). Equilibrium investment strategy for DC pension plan with default risk and return of premiums clauses under CEV model. Insurance, 72, 6-20.

[22] Osu, B. O., Akpanibah, E. E.\& Olunkwa, C. (2018). Mean-Variance Optimization of portfolios with return of premium clauses in a DC pension plan with multiple contributors under constant elasticity of variance model. textitInternational Journal of Mathematics and Computer Science, $12,85-90$.

[23] He, Y. \& Chen, P. (2020). Optimal Investment Strategy under the CEV Model with Stochastic Interest Rate, Mathematical Problems in Engineering, 2020, 1-11

[24] Akpanibah E. E. \& U. O. Ini (2020) Portfolio Strategy for an Investor with Logarithm Utility and Stochastic Interest Rate under Constant Elasticity of Variance Model., Journal of the Nigerian Society of Physical Sciences, 2(3), 186-196.

[25] Ihedioha, S. A., Danat, N. T.\& Buba, A. (2020). Investor's Optimal Strategy with and Without Transaction Cost Under Ornstein-Uhlenbeck and Constant Elasticity of Variance (CEV) Models via Exponential Utility Maximization. Pure and Applied Mathematics Journal, 9(3), $55-63$.

[26] Akpanibah, E. E. \& Ini, U. O. (2021). An Investor's Investment Plan with Stochastic Interest Rate under the CEV Model and the O-U Process. Journal of the Nigerian Society of Physical Sciences, 3(3), 239-249. 
[27] Zhao, H. \& Rong, X. (2012). Portfolio selection problem with multiple risky assets under the constant elasticity of variance model. Mathematics and Economics, 50, 179-190.

[28] Cox, J.C. (1996). The constant elasticity of variance option pricing model. The Journal of Portfolio Management, 23, 15-17. 